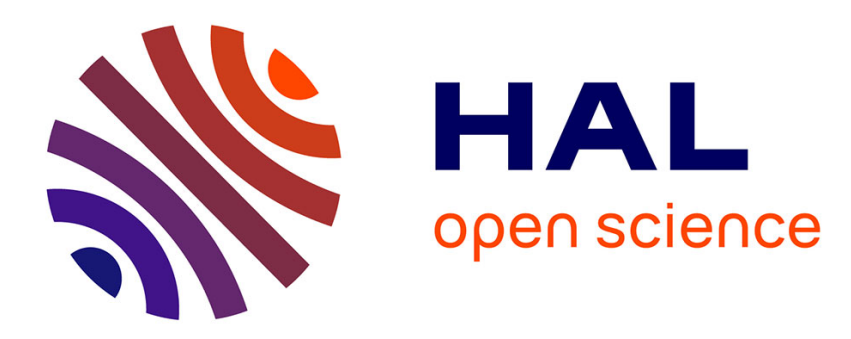

\title{
Effets Raman stimulés dans la calcite
}

\author{
Gaetan Bisson, G. Mayer
}

\section{To cite this version:}

Gaetan Bisson, G. Mayer. Effets Raman stimulés dans la calcite. Journal de Physique, 1968, 29 (1), pp.97-110. 10.1051/jphys:0196800290109700 . jpa-00206625

\section{HAL Id: jpa-00206625 https://hal.science/jpa-00206625}

Submitted on 1 Jan 1968

HAL is a multi-disciplinary open access archive for the deposit and dissemination of scientific research documents, whether they are published or not. The documents may come from teaching and research institutions in France or abroad, or from public or private research centers.
L'archive ouverte pluridisciplinaire HAL, est destinée au dépôt et à la diffusion de documents scientifiques de niveau recherche, publiés ou non, émanant des établissements d'enseignement et de recherche français ou étrangers, des laboratoires publics ou privés. 


\title{
EFFETS RAMAN STIMULÉS DANS LA GALGITE
}

\author{
Par G. BISSON et G. MAYER. \\ Département de Recherches Électro-optiques de la G.S.F., Domaine de Corbeville, 9I-Orsay.
}

(Reçu le 28 juillet 1967.)

\begin{abstract}
Résumé. - Nous décrivons des expériences conçues pour vérifier dans la calcite la théorie des effets Raman stimulés du premier ordre.

Cette théorie prévoit pour l'onde Stokes d'intensité $I_{\mathrm{S}}$ l'existence d'un indice de gain $\beta$ ne dépendant que de l'intensité excitatrice $I_{\mathrm{L}}$ tant que $I_{\mathrm{S}} \ll I_{\mathrm{L}}$.

Toutes les expériences confirment ce point fondamental, mais, quantitativement, la relation observée entre $\beta$ et l'intensité moyenne $\bar{I}_{\mathrm{L}}$ n'est pas celle qu'on attendait.

Pour concilier théorie et expériences, la présence dans le faisceau laser de zones étroites où l'intensité $I_{\mathrm{L}}$ atteindrait $10 \vec{I}_{\mathrm{L}}$ serait une condition nécessaire.

L'observation directe du faisceau ne nous a montré que des fluctuations $I_{\mathrm{L}}<4 \bar{I}_{\mathrm{L}}$.

De plus, la mesure directe de la fonction $\beta\left(I_{\mathrm{L}}\right)$ dans ces zones de plus grande intensité ne révèle pas la forme linéaire attendue.
\end{abstract}

Abstract. - Paying due attention to the inhomogeneities of the exciting laser beams, experiments have been designed to check in calcite the theory of first order stimulated Raman effects.

For the Stokes wave of intensity $I_{\mathrm{s}}$, this theory predicts an index of gain $\beta$ dependent only on the exciting intensity $I_{\mathrm{L}}$, provided that $I_{\mathrm{S}} \ll I_{\mathrm{L}}$.

All the experiments support this fundamental point, but the numerical relation observed between $\beta$ and the average intensity $\bar{I}_{\mathrm{L}}$ is not the one expected.

To get an agreement between theory and experiment, we have to assume the existence in the laser beams of narrow areas where the intensity $I_{\mathrm{L}}$ would reach $10 \bar{I}_{\mathrm{L}}$ from time to time.

Direct observations of laser beams with resolving powers of $10^{-2} \mathrm{~cm}$ and $10^{-9} \mathrm{~s}$ have not shown fluctuations of $I_{\mathrm{L}}$ larger than $4 \bar{I}_{\mathrm{L}}$. No self-focusing effects on the exciting light have been observed in calcite.

Furthermore, direct measurements of the function $\beta\left(I_{\mathrm{L}}\right)$ in the areas of highest intensity $I_{\mathrm{L}}$ do not show the expected linear form.

La théorie de Hellwarth montre clairement la relation numérique qui associe les effets Raman spontanés et stimulés. Cette relation devient particulièrement simple quand on étudie un effet relatif à une vibration mécanique de fréquence $\omega_{\mathrm{v}}$ à une température $T$ telle que $\hbar \omega_{\mathrm{v}}>k T$.

Nous avons étudié les effets Raman liés à la vibration symétrique des atomes d'oxygène des groupes $\mathrm{CO}_{3}$ de la calcite dans des plans perpendiculaires à l'axe optique; $\omega_{\mathrm{v}}=1086 \mathrm{~cm}^{-1}$.

Les mesures à température ordinaire de l'intensité et de la largeur de raie $\Delta \omega_{\mathrm{s}}$ de l'effet spontané permettent de prévoir qu'en présence d'une intensité excitatrice $I_{\mathrm{L}}$ à la fréquence $\omega_{\mathrm{L}}\left(\omega_{\mathrm{L}} \simeq 1,4 \times 10^{4} \mathrm{~cm}^{-1}\right)$, il existera autour de la fréquence $\omega_{\mathrm{S}}=\omega_{\mathrm{L}}-\omega_{\mathrm{v}}$ un indice de gain positif; si l'onde $\omega_{\mathrm{L}}$ occupe un intervalle spectral nettement inférieur à $\Delta \omega_{\mathrm{S}}$, on aura :

$$
\begin{gathered}
\beta\left(\omega_{\mathrm{S}}\right)=b\left(\omega_{\mathrm{S}}\right) I_{\mathrm{L}} \mathrm{cm}^{-1} ; \\
b\left(\omega_{\mathrm{S}}\right)=4,4 \times 10^{-3} \mathrm{~cm} \cdot \mathrm{MW}^{-1} .
\end{gathered}
$$

Cette relation est valable quand l'onde $\omega_{\mathrm{L}}$ et l'onde $\omega_{\mathrm{S}}$ ont une direction de polarisation rectiligne commune qui coïncide avec la direction de polarisation ordinaire de la calcite. Elle est valable aussi quand les ondes $\omega_{\mathrm{L}}$ et $\omega_{\mathrm{S}}$ ont une polarisation circulaire de même sens, la direction de propagation commune étant celle de l'axe optique de la calcite.

Les faisceaux lasers. - L'équation (1) montre que seuls les lasers déclenchés permettent d'obtenir des intensités $I_{\mathrm{L}}$ associées à des indices de gain facilement observables. Par exemple, pour $I_{\mathrm{L}}=100 \mathrm{MW} . \mathrm{cm}^{-2}$, $\beta\left(\omega_{\mathrm{s}}\right)=0,44 \mathrm{~cm}^{-1}$.

Presque toutes nos expériences ont été faites avec des lasers à rubis, déclenchés par des verres à absorption saturable, comportant parfois un étage amplificateur; la durée de leur impulsion est de l'ordre de $2,5 \times 10^{-8} \mathrm{~s}$; leur largeur spectrale était toujours inférieure à $10^{9} \mathrm{~s}^{-1}$.

Nous avons vérifié que des effets stimulés forts 
pouvaient aussi être obtenus avec un laser à verre de néodyme déclenché par un prisme tournant.

La divergence des faisceaux des lasers à rubis allait de 3 à 7 milliradians selon les appareils utilisés.

Nous avons étudié la structure des faisceaux en mesurant simultanément les puissances $P_{\mathrm{I}}$ et $p_{\mathrm{L}}$ en amont et en aval de petits diaphragmes avec une résolution temporelle de $2 \times 10^{-9} \mathrm{~s}$. La figure 1
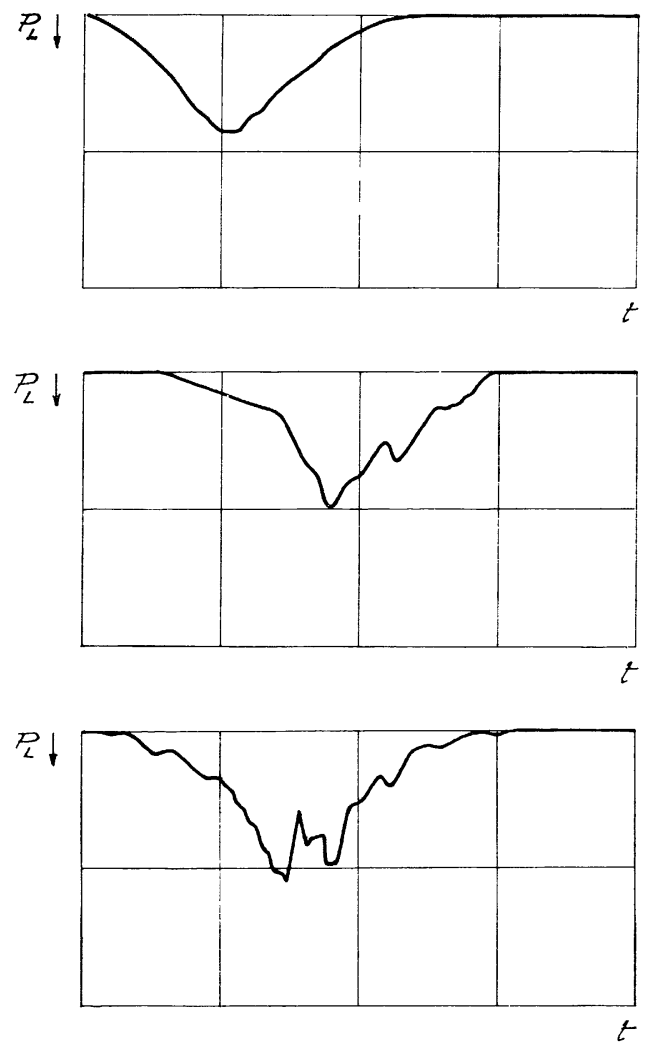

FIG. 1. - Puissance $p_{\mathrm{L}}$ du faisceau laser transmis par des diaphragmes divers :

$p_{\mathrm{L}}$ est mesurée avec une résolution temporelle d'environ $2 \times 10^{-9} \mathrm{~s}$. De haut en bas : $\varphi=3 \mathrm{~mm}$; $\varphi=0,4 \mathrm{~mm} ; \varphi=0,2 \mathrm{~mm}$, ordonnées arbitraires.

montre ainsi $p_{\mathrm{L}}(t)$ à travers des trous ronds de différents diamètres $d$.

Pour le plus grand diaphragme (faisceau presque entier), la modulation temporelle est faible. Pour $d=4 \times 10^{-2} \mathrm{~cm}$, elle est nettement marquée. Pour $d=2 \times 10^{-2} \mathrm{~cm}$, elle est plus profonde encore; le temps de corrélation est de l'ordre de 2 à $3 \times 10^{-9} \mathrm{~s}$. Le diamètre des aires de cohérence est donc inférieur à $4 \times 10^{-2} \mathrm{~cm}$. Estimons-le à $2 \times 10^{-2} \mathrm{~cm}$ et soit $s$ la surface correspondante. Sur la section $S\left(S \simeq 10^{-1} \mathrm{~cm}^{2}\right)$ du faisceau tout entier, pendant la durée de l'impulsion laser, il y a donc environ 1500 « pointsinstants » à considérer.

L'intensité $i_{\mathrm{L}}$ en un de ces «points-instants » peut dépasser la valeur moyenne $\bar{I}_{\mathrm{L}}$ d'un facteur assez différent de l'unité.

L'examen d'une centaine de photographies (correspondant à environ 700 《 points-instants ») nous a montré sur chaque photographie :

$$
p_{\mathrm{L}}(t)-\bar{p}_{\mathrm{L}}<\bar{p}_{\mathrm{L}} \quad(\mathrm{a})
$$

$\bar{p}_{\mathrm{L}}$ désigne la valeur crête observée avec une résolution de $10^{-8} \mathrm{~s}$.

D'autre part, l'ensemble des photographies montre :

$$
\bar{p}_{\mathrm{L}}-\overline{\bar{p}}_{\mathrm{L}}<\overline{\bar{p}}_{\mathrm{L}} \quad(\mathrm{b}) \quad \bar{p}_{\mathrm{L}}=\frac{s}{S} \bar{P}_{\mathrm{L}}
$$

$\overline{\bar{p}}_{\mathrm{L}}$ désigne la moyenne de $\bar{p}_{\mathrm{L}}$ sur toutes les photographies.

Les deux inégalités mises bout à bout montrent :

$$
p_{\mathrm{L}}(t)<2 \bar{p}_{\mathrm{L}}<4 \overline{\bar{p}}_{\mathrm{L}}
$$

L'égalité (c) permet de traduire les puissances en intensité :

$$
i_{\mathrm{L}}(t)<4 \bar{I}_{\mathrm{L}}
$$

A propos des «mesures de seuil », nous décrivons plus loin les expériences qui précisent cette inégalité (cf. page 107).

Nous avons vérifié, en disposant en amont du diaphragme un tube vide, que la traversée de l'atmosphère ne créait pas de singularité dans le faisceau.

Influence de la cavité résonnante. - Dans la première série d'expériences sur la calcite, nous avons cru pouvoir étudier le milieu amplificateur à la fréquence Stokes $\omega_{\mathrm{s}}$ 《sans cavité résonnante » autour de lui.

Pour cela, nous utilisions des échantillons de formes assez biscornues. De nombreuses observations visuelles au convertisseur d'images $\left(\lambda_{\mathrm{S}} \simeq 0,75 \mu\right)$ de la structure spatiale de l'onde Stokes nous ont convaincus que c'était toujours une certaine proportion de «feedback» souvent faible, toujours incontrôlée qui permettait l'installation de modes énergiques d'oscillation. L'apparition de défauts permanents dans la calcite qui se produit pour une intensité moyenne d'irradiation d'environ $150 \mathrm{MW} . \mathrm{cm}^{-2}$ interrompait souvent les expériences.

Il nous a paru préférable d'établir délibérément une cavité résonnante simple et contrôlable autour de la calcite pour continuer ces études. Nous avons donc muni nos échantillons d'une paire de faces planes parallèles polies par lesquelles entre et sort le faisceau excitateur.

Dans certains échantillons, les faces polies sont taillées perpendiculaires à l'axe optique; dans d'autres, elles lui sont parallèles. Cette disposition est destinée à favoriser les modes d'oscillation associés à une propagation le long de la normale aux faces polies.

L'existence de ces modes est rendue vraisemblable par une expérience très simple : même lorsque l'axe du faisceau excitateur ne coïncide pas avec la normale 
aux faces, l'émission Stokes intense a bien lieu dans la direction de celle-ci avec une intensité égale en avant et en arrière ( fig. 2).

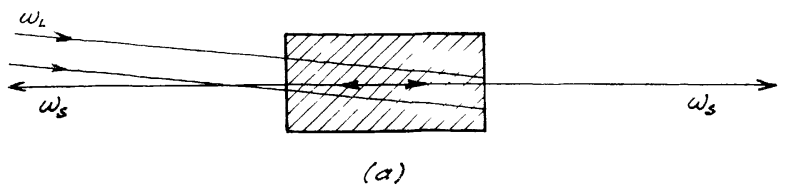

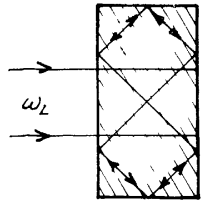

(b)

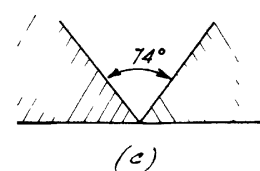

(c)

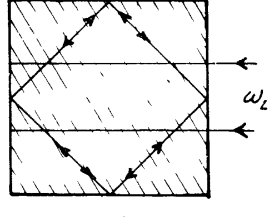

(d)
Frg. 2. - Modes d'oscillation de cristaux à faces polies :

(a) : Mode longitudinal. - (b) et (d) : Modes bénéficiant de réflexions totales. - (c) : La partie hachurée montre le domaine angulaire des rayons subissant la réflexion totale quand le spath est dans l'air.

Là est l'avantage principal des expériences à feedback contrôlé : on sait où disposer les appareils de mesure qui guetteront l'apparition des oscillations.

Nos échantillons ont des longueurs allant de 0,2 à $8 \mathrm{~cm}$ et des sections carrées de $1 \mathrm{~cm}$ de côté. Le diamètre $\mathrm{du}$ faisceau excitateur est en général de l'ordre de $0,3 \mathrm{~cm}$.

Dans les échantillons courts, d'autres modes apparaissent si on ne prend pas la précaution de dépolir les faces latérales. Dans un cube, par exemple, le mode schématisé sur la figure 2 bénéficie de quatre réflexions totales. Dans un demi-cube (notre cristal de $0,5 \mathrm{~cm}$ de long), on peut, même en se restreignant au plan de la figure, trouver des modes qui ne subissent que des réflexions totales car l'angle limite d'incidence vaut $37^{\circ}$.

On supprime ces modes latéraux en immergeant le cristal dans l'eau; l'angle limite remonte alors à $53^{\circ}$.

En diminuant le diamètre de la zone excitée, on défavorise les modes latéraux par rapport aux modes longitudinaux que nous avons surtout étudiés.

Nos études sont guidées par le schéma le plus simple : durant sa brève excitation par le faisceau laser, la calcite est rendue amplificatrice à la fréquence $\omega_{\mathrm{s}}$. L'énergie d'un des modes d'oscillation définis par les miroirs qui terminent le cristal pourra croître aussi longtemps que le pouvoir amplificateur surmontera les pertes relatives à ce mode.

Transition entre régimes spontané et stimulé. A. LES RÈGLES DU JEU. - Quand $I_{\mathrm{L}}$ est suffisamment basse, la puissance Stokes $P_{\mathrm{S}}$ est proportionnelle à $I_{\mathrm{L}}$.

Si $I_{\mathrm{L}}$ devient assez forte pour que l'indice de gain $\beta=b(\omega) I_{\mathrm{L}}$ multiplié par la longueur du cristal ne soit plus négligeable devant l'unité, $P_{\mathrm{S}}$ variera plus vite que $I_{\mathrm{L}}$.

Dans les conditions difficiles à réaliser que nous allons énumérer, la quantité de photons Stokes trans-

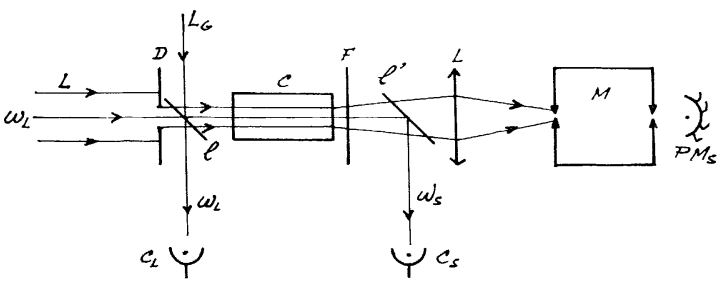

FIG. 3. - Dispositif de mesure des effets Raman :

D : Diaphragmes. - 1, 1' : Lames transparentes. c : Calcite. - F : Filtre opaque à $\omega_{\mathrm{L}}$. - L : Lentille. - $\mathrm{C}_{\mathrm{L}}, \mathrm{C}_{\mathrm{S}}$ : Cellules photoélectriques. - M : Monochromateur. - $\mathrm{PM}_{\mathrm{S}}$ : Photomultiplicateur. Un faisceau de laser à gaz $L_{\mathrm{G}}$ matérialise pour les réglages le trajet du faisceau laser L.

mise par le monochromateur au détecteur (fig. 3) vaudra par unité de temps, en l'absence de feedback :

$$
\dot{\varphi}_{\mathrm{S}}(\omega)=b(\omega) I_{\mathrm{L}} \frac{z}{\lambda_{\mathrm{S}}^{2}} \frac{\mathrm{e}^{\left[b(\omega) I_{\mathrm{L}}-\alpha\right] l}-1}{\left[b(\omega) I_{\mathrm{L}}-\alpha\right] l}
$$

$\alpha$ est l'indice d'une éventuelle absorption; $l$ est la longueur excitée de l'échantillon; $\lambda_{\mathrm{s}}$ est la longueur d'onde Stokes; $z$ désigne le produit des coordonnées expérimentales s.l. $\Delta \Omega . T . \delta \omega$.

$\Delta \Omega$ est l'angle solide vu par le détecteur à travers le monochromateur dont la transmission est $T$ et la largeur spectrale $\delta \omega$.

$\Delta \Omega$ doit être tout entier compris dans l'angle défini par les paramètres $s$ et $l$ de la zone excitée.

$\delta \omega$ doit être petite devant la largeur spontanée $\Delta \omega_{\mathrm{S}}$. De plus, $I_{\mathrm{L}}$ doit être la même dans tout le volume excité. On ne peut lui demander d'être constante dans le temps puisqu'il s'agit de l'impulsion d'un laser déclenché, mais l'équation (2) ne sera vérifiable expérimentalement que si le temps de résolution $\tau \mathrm{du}$ détecteur Stokes est nettement inférieur à la durée de l'impulsion laser.

C'est en comparant les $\dot{\varphi}_{\mathrm{s}}$ émis par des échantillons de diverses longueurs $l$ avec la même $I_{\mathrm{L}}$ que l'on peut obtenir la valeur de $\alpha$.

C'est évidemment au pic de la raie spontanée pour $\omega=\omega_{\mathrm{S}}=\omega_{\mathrm{L}}-\omega_{\mathrm{v}}$ que l'effet de l'indice de gain $b(\omega) I_{\mathrm{L}}$ risque d'être le plus net.

La courbe $\varphi_{\mathrm{S}}\left(I_{\mathrm{L}}\right)$ commence par une partie rectiligne que nous appelons la droite spontanée; pour des $I_{\mathrm{L}}$ plus grandes, un facteur multiplicateur élève $\dot{\varphi}_{\mathrm{S}}$ au-dessus de la droite spontanée.

Si les conditions de bonnes résolutions spectrale et temporelle ne sont pas satisfaites, le facteur multiplicateur observé sera moins grand.

Plaçons-nous par exemple dans le cas où $\alpha l$ est négligeable, et où la résolution temporelle est bonne. 
Si la résolution spectrale est bonne $\left(\delta \omega<\Delta \omega_{\mathrm{S}}\right)$, on observe des facteurs multiplicatifs de $6,4=\frac{\mathrm{e}^{3}-1}{3}$ pour $\beta\left(\omega_{\mathrm{s}}\right) l=3$ et de 1,72 pour $\beta\left(\omega_{\mathrm{s}}\right) l=1$. Au contraire, avec $\delta \omega>\Delta \omega_{\mathrm{s}}$, on observerait respectivement des facteurs de 3,8 et 1,47 . Une mauvaise résolution temporelle apporte aussi un affaiblissement du facteur mesuré.

Au contraire, si la condition d'homogénéité spatiale de $I_{\mathrm{L}}$ n'est pas satisfaite, le facteur mesuré s'en trouvera augmenté, la moyenne de l'exponentielle étant plus grande que l'exponentielle de la moyenne. Des fluctuations temporelles trop rapides pour être analysées auront le même effet.

Heureusement, le coefficient $b(\omega)$ peut être mesuré sans les précautions nécessaires à la vérification de la partie courbe de la fonction $\dot{\varphi}_{\mathrm{s}}(\omega)$. Il suffit d'opérer à un niveau $I_{\mathrm{L}}$ suffisamment bas pour que $b(\omega) I_{\mathrm{L}} l \ll 1$. Les conditions d'homogénéité spatiale et de résolution temporelle ne sont plus nécessaires puisque $\dot{\varphi}_{\mathrm{S}}$ est simplement proportionnelle à $I_{\mathrm{L}}$.

La résolution spectrale est elle aussi superflue si la forme de la raie spontanée a été mesurée indépendamment.

En présence de feedback, le flux $\dot{\varphi}_{\mathrm{S}}(\omega)$ émis devient supérieur au $\dot{\varphi}_{\mathrm{S}}(\omega)$ donné par l'équation (2). Si, par exemple, il est dû au pouvoir réflecteur $R$ des faces terminales, on aura :

$$
\dot{\Phi}_{\mathrm{S}}(\omega)=\dot{\varphi}_{\mathrm{S}}(\omega) \frac{1}{1-R \mathrm{e}^{\left[b(\omega) I_{\mathrm{L}}-\alpha\right] l}}
$$

tant que $R \mathrm{e}^{\left[b(\omega) I_{\mathrm{L}}-\alpha\right] l}<1$ et le système entrera en oscillations si $\operatorname{Re}^{\left[b(\omega) I_{\mathrm{L},}-\alpha\right] l}>1$.

B. RÉsultats expérimentaux. - L'évolution de la puissance Stokes $P_{\mathrm{S}}$ en fonction de l'intensité moyenne $\bar{I}_{\mathrm{L}}$ a été observée avec le dispositif de la figure 3. Le photomultiplicateur PM associé au monochromateur est assez sensible pour mesurer l'émission Raman spontanée. La cellule $C_{\mathrm{S}}$ ne peut mesurer que les $P_{\mathrm{S}}$ supérieures à 10 watts; son ouverture angulaire de $10^{-3}$ stér. assure une bonne réception de l'énergie émise par les modes longitudinaux. La cellule $C_{\mathrm{I}}$ mesure la valeur moyenne $\bar{I}_{\mathrm{L}}$ avec une résolution de $6 \times 10^{-9} \mathrm{~s}$. Dans l'expérience décrite (page 107), la résolution atteint $2 \times 10^{-9} \mathrm{~s}$.

Nous avons dû opérer dans la mauvaise situation $\delta \omega>\Delta \omega_{\mathrm{s}}$ pour deux raisons :

- si on ferme les fentes du monochromateur, on agit simultanément sur deux coordonnées expérimentales, la largeur spectrale $\delta \omega$ et l'angle solide $\Delta \Omega$, et le nombre de photons parvenant au PM tombe vite en dessous du niveau critique;

- d'autre part, la largeur de la raie Raman ne vaut que $1,2 \mathrm{~cm}^{-1}$ et il suffit d'une variation de quelques degrés du rubis $\left(\partial \omega_{\mathrm{L}} / \partial T=0,14 \mathrm{~cm}^{-1}{ }^{\circ} \mathrm{C}^{-1}\right)$ pour s'écarter du pic.

La résolution temporelle du PM est de l'ordre de $10^{-8} \mathrm{~s}$.
Etude du pied de $P_{\mathrm{s}}\left(\bar{I}_{\mathrm{L}}\right)$. - L'étude des droites spontanées (partie rectiligne de $P_{\mathrm{S}}\left(\bar{I}_{\mathrm{L}}\right)$ d'échantillons de diverses longueurs nous a montré que l'atténuation $\alpha$ était négligeable autour de $\omega_{\mathrm{s}}$. Leur pente donne $b\left(\omega_{\mathrm{s}}\right)=4,4 \times 10^{-3} \mathrm{~cm} . \mathrm{MW}^{-1}$.

Dans un échantillon de $1 \mathrm{~cm}$, par exemple, c'est pour $I_{\mathrm{L}} \simeq 225 \mathrm{MW} . \mathrm{cm}^{-2}$ que le facteur multiplicatif de l'équation (2) atteindra 1,7 et que la courbe $P_{\mathrm{S}}\left(\bar{I}_{\mathrm{L}}\right)$ devra montrer une franche courbure dans nos conditions d'observation.

Or, pour un tel échantillon, la courbe $P_{\mathrm{S}}\left(\bar{I}_{\mathrm{L}}\right)$ est effectivement linéaire jusqu'aux $\overline{I_{\mathrm{L}}}$ voisins de $60 \mathrm{MW} \cdot \mathrm{cm}^{-2}$, ce qui est parfaitement normal, mais elle saute alors de plusieurs ordres de grandeur parce que des oscillations prennent naissance entre les faces terminales, et cela est tout à fait surprenant.

En effet, pour $\bar{I}_{\mathrm{L}}=60 \mathrm{MW} \cdot \mathrm{cm}^{-2}$, le gain prédit à partir de la pente des droites spontanées vaut $\mathrm{e}^{\mathbf{0}, 26}$ et les oscillations prouvent que, par endroit et par instant, ce gain a atteint au moins la valeur $\mathrm{e}^{2,8}$ nécessaire pour compenser la perte par transmission de $94 \%$ sur chaque face terminale.

Ce désaccord numérique suggère qu'il pourrait exister à l'intérieur du cristal des zones allant d'une face à l'autre et contenant leur normale où l'intensité $I_{\mathrm{L}}$ vaut au moins dix fois la valeur moyenne $\bar{I}_{\mathrm{L}}$.

Quelques données numériques vont préciser dans quelles conditions se fait la détection du démarrage des oscillations.

Un cristal long de $1 \mathrm{~cm}$ éclairé sur une surface de $0,1 \mathrm{~cm}^{2}$ par une intensité moyenne de $50 \mathrm{MW} . \mathrm{cm}^{-2}$ envoie dans l'angle solide de $3 \times 10^{-5}$ stér. du monochromateur $\left(\theta_{\perp}=1,5 \times 10^{-3} \mathrm{rd} ; \theta_{/ /}=2 \times 10^{-2} \mathrm{rd}\right)$ environ 4 microwatts de rayonnement spontané. Pendant les $10^{-8} \mathrm{~s}$ du temps de résolution du PM, cela fait $4 \times 10^{-7}$ ergs $\left(\simeq 2 \times 10^{5}\right.$ photons $)$.

Des expériences décrites plus loin suggèrent qu'audessus du seuil l'énergie Stokes est émise par des zones d'environ $10^{-4} \mathrm{~cm}^{2}$ et que l'intensité $I_{\mathrm{S}}$ qui y règne peut approcher $I_{\mathrm{L}}$ si le seuil d'oscillation reste franchi assez longtemps.

Mais l'oscillation est détectée beaucoup plus tôt. En effet, si on suppose que son diagramme de rayonnement reste constamment celui d'une tache circulaire de $10^{-2} \mathrm{~cm}$ de diamètre, la fente du monochromateur acceptera le quart de son émission; quand $I_{\mathrm{S}}$ atteint $0,2 \mathrm{~W} \cdot \mathrm{cm}^{-2}$ dans une telle zone, la puissance qu'elle envoie au PM est égale à celle du rayonnement spontané envoyé par le reste du cristal. Quand $I_{\mathrm{S}}$ atteint quelques dizaines de $\mathrm{kW} . \mathrm{cm}^{-2}$, le détecteur $C_{\mathrm{s}}$ moins sensible commence à répondre.

Avec le même dispositif expérimental, nous avons $\mathrm{pu}$ observer dans les cuves de $10 \mathrm{~cm}$ de benzène munies de faces obliques une courbure de la fonction $P_{\mathrm{S}}\left(\bar{I}_{\mathrm{L}}\right)$ conforme à l'équation (2) dans tout le domaine de $I_{\mathrm{L}}$ où l'autopiégeage n'intervient pas encore. Mais, dans la calcite, l'observation de $P_{\mathrm{S}}\left(\bar{I}_{\mathrm{L}}\right)$ 
faite sur des cristaux de $1 \mathrm{~cm}$ avec leurs faces dans l'air (pouvoir réflecteur $R=6 \%$ ) ou dans l'eau $(R \simeq 1 \%)$ a toujours montré une partie strictement linéaire (la droite spontanée) interrompue par des sauts de plusieurs ordres de grandeur.

Dans un domaine de $\bar{I}_{\mathrm{L}}$ d'étendue relative voisine de $20 \%$ que nous appellerons seuil $\overline{I_{\mathrm{L}}^{0}}$, on obtient (fig. 4) :

- soit des points alignés sur la droite spontanée définie par les mesures faites à bas niveau de $\bar{I}_{\mathrm{L}}$;

- soit des points $P_{\mathrm{s}}$ situés à plusieurs ordres de grandeurs au-dessus de la droite spontanée;

- soit exceptionnellement (environ une fois sur cent) des points à moins d'un ordre de grandeur audessus de cette droite.

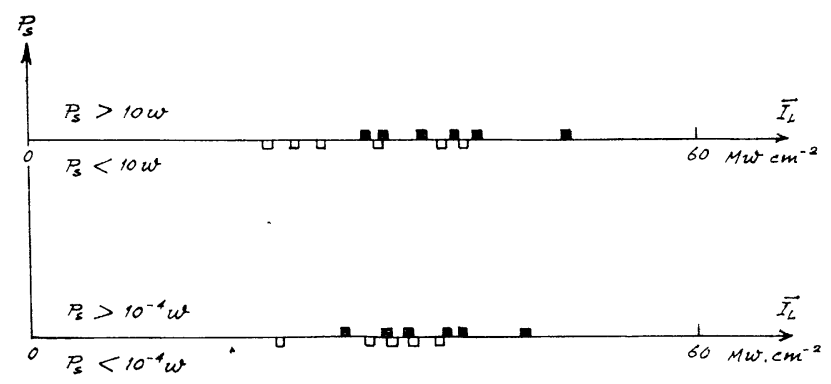

FIG. 4. - Détermination expérimentale des seuils :

Observation simultanée de l'émission Raman par une cellule (haut) et un photomultiplicateur (bas). Cristal de $4 \mathrm{~cm}$; lumière convergente.

Le seuil défini ( $f i g .4$ ) par la cellule $C_{\mathrm{S}}$ est légèrement plus élevé que le seuil défini par le PM, mais ne s'en écarte pas de $10 \%$. La courbe des seuils en fonction de la longueur des échantillons que nous présentons plus loin montre que ce sont les cristaux longs qui montrent au seuil le plus fort rayonnement spontané. D'autre part, des faces terminales faiblement réfléchissantes augmentent le seuil en décourageant les oscillations.

En conséquence, nous avons recherché avec un soin particulier la courbure prévue par l'équation (2) dans un cristal de $8 \mathrm{~cm}$ noyé dans l'eau. En n'introduisant dans le bilan des pertes et des gains que les pertes par transmission des faces, on trouve que les oscillations deviennent possibles quand le gain dépasse $\mathrm{e}^{\mathbf{4 , 6}}$. La courbe expérimentale $P_{\mathrm{S}}\left(\bar{I}_{\mathrm{L}}\right)$ est néanmoins restée linéaire jusqu'au seuil $I_{\mathrm{L}}^{0} \simeq 25 \mathrm{MW} . \mathrm{cm}^{-2}$ (comme dans le cas de la figure 5). La dispersion des points expérimentaux autour de la droite spontanée n'excède pas $\pm 10 \%$ en valeur relative. Si la moyenne de $\beta l$ avait été plus grande que 0,7 , le facteur $\left(e^{\beta l}-1\right) / \beta l$ aurait dépassé 1,4 et nous l'aurions détecté. Il y a deux surprises :

- L'absence de courbure pour $I_{\mathrm{I}}<I_{\mathrm{L}}^{0}$ :

Avec la valeur observée du seuil $I_{\mathrm{L}}^{0}$, l'équation (1) donne $\beta l=0,88$. La courbe expérimentale aurait dû

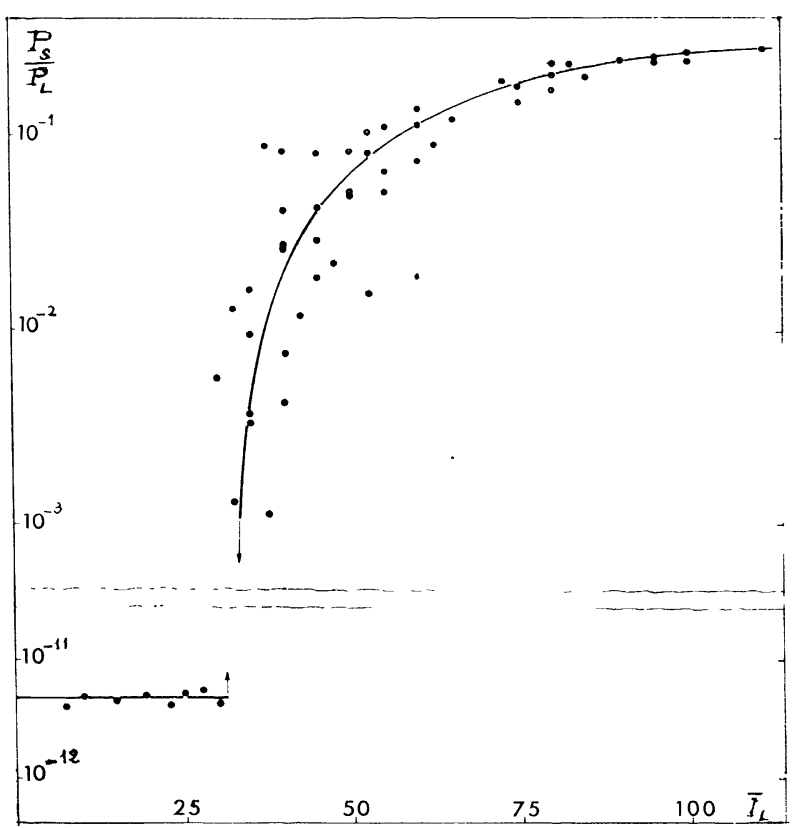

FIG. 5. - Rapport de la puissance crête Raman $P_{\mathrm{S}}$ émise en avant à la puissance crête $P_{\mathrm{L}}$ d'excitation en fonction de l'intensité crête moyenne $\overline{I_{\mathrm{L}}}$ :

$\bar{I}_{\mathrm{L}}$ : mesurée en $\mathrm{MW} . \mathrm{cm}^{-2}$ sur un diaphragme de $3 \mathrm{~mm}$. - Échantillon de $4 \mathrm{~cm}$. - Lumière convergente. - Partie inférieure : observation de $P_{\mathrm{S}}$ avec une résolution de $10^{-8} \mathrm{~s}$. - Partie supérieure : observation de $P_{\mathrm{S}}$ avec une résolution de $2 \times 10^{-9} \mathrm{~s}$. $\bar{P}_{\mathrm{L}}$ est mesurée avec une résolution de $2 \times 10^{-9} \mathrm{~s}$.

se détacher de la droite spontanée. Ce point n'est pas grave; bien que l'appareil ait été «calibré » par l'étude préalable du benzène, nous avons pu surestimer $b(\omega)$;

- La présence d'oscillations pour $\bar{I}_{\mathrm{L}} \geqslant \bar{I}_{\mathrm{L}}^{0}$ :

L'observation dans le même dispositif de la linéarité du début de $P_{\mathrm{S}}\left(\bar{I}_{\mathrm{L}}\right)$ qui indique $b\left(\omega_{\mathrm{S}}\right) \bar{I}_{\mathrm{L}} l<0,7$, interrompue par des oscillations qui prouvent que localement $b\left(\omega_{\mathrm{S}}\right) I_{\mathrm{L}} l>4,6$ est une indication plus solide, car la validité simultanée de ces deux inéquations est indépendante de la qualité de nos étalonnages.

Plafond de $P_{\mathrm{S}}\left(\bar{I}_{\mathrm{L}}\right)$. - Ces résultats suggèrent que les oscillations démarrent sur des fluctuations de $I_{\mathrm{L}}$ assez éloignées de la valeur moyenne mesurée $\bar{I}_{\mathrm{L}}$.

Nous avons déjà décrit plus haut des mesures de l'hétérogénéité spatio-temporelle des faisceaux excitateurs; elles ne montrent pas de si fortes fluctuations. Mais, quelles que soient leurs amplitudes, leurs formes et leurs durées, quelle que soit la signification physique du seuil d'oscillation, nous sommes obligés de faire appel à ces hétérogénéités pour expliquer l'absence de courbure au pied de $P_{\mathrm{s}}\left(\bar{I}_{\mathrm{L}}\right)$ et la forme de la partie 


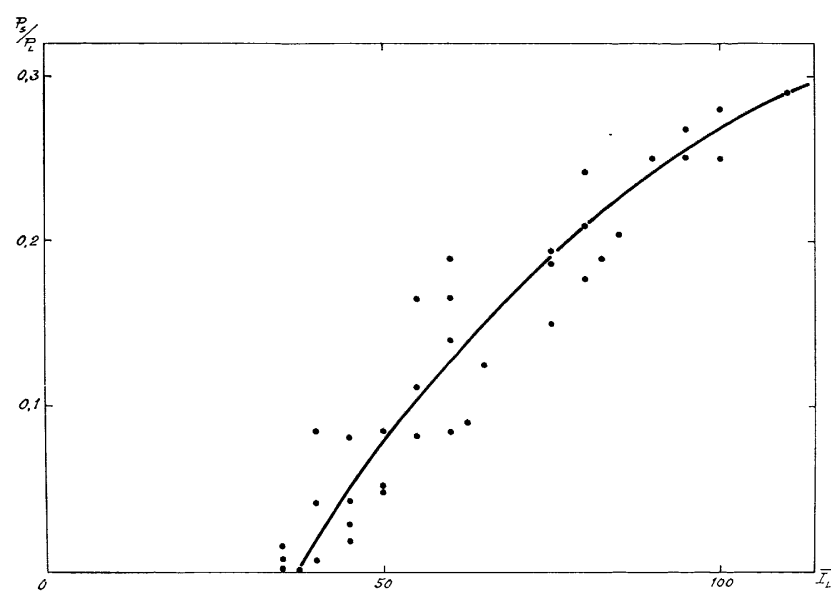

FIG. 5 a. - Haut de la courbe de la figure 5 présentée en échelle linéaire.

supérieure de la courbe ( $f i g .5$ et 5 a). Comment expliquer autrement que les oscillations ne s'allument pas simultanément sur toute la section excitée $S$ pour la même valeur repérée de $\bar{I}_{\mathrm{L}}$ ? Les courbes de la figure 5 sont relatives à un échantillon de $4 \mathrm{~cm}$. Les valeurs de $P_{\mathrm{s}}$ supérieures à $1 \mathrm{~kW}$ ont été mesurées avec un temps de résolution de $2 \times 10^{-9}$ s. Si les hétérogénéités n'existaient pas, $P_{\mathrm{S}}\left(\bar{I}_{\mathrm{L}}\right)$ serait plus raide encore.

On voudrait déduire de ces courbes des indications sur la loi statistique de distribution des intensités dans le faisceau excitateur. Pour cela, il faut connaître la cinétique de croissance des oscillations une fois le seuil localement franchi à un instant donné. Il faut aussi savoir vers quel niveau $I_{\mathrm{S}}^{\mathrm{m}}$ tend $I_{\mathrm{S}}$ quand $I_{\mathrm{L}}$ reste assez longtemps au-dessus du seuil local d'oscillation. G'est le rapport $I_{\mathrm{S}}^{\mathrm{m}} / I_{\mathrm{L}}$ qui fixe le plafond de $P_{\mathrm{S}} / P_{\mathrm{L}}$ quand tout le volume excité fonctionne en oscillations saturées. G'est de ce plafond et non de la valeur unité qu'il faut redescendre pour atteindre la loi statistique recherchée.

Une expérience décrite plus loin suggère $I_{\mathrm{S}}^{\mathrm{m}} / I_{\mathrm{L}} \simeq 0,2$; rappelons que la même $I_{\mathrm{S}}$ est émise en avant et en arrière. L'incertitude relative à ce plafond et l'impossibilité pratique de prolonger les courbes $P_{\mathrm{S}}\left(P_{\mathrm{L}}\right)$ nous empêchent d'utiliser avec précision le haut de ces courbes, mais on peut remarquer deux points :

- Pour $\quad \bar{I}_{\mathrm{L}}=3 \overline{I_{\mathrm{L}}^{0}}, \quad P_{\mathrm{S}}$ est déjà proche de $P_{\mathrm{L}}$;

- La figure 5 a ne donne pourtant pas l'impression d'être près d'atteindre son plafond horizontal.

Rappelons que l'analyse du pied des courbes suggère un facteur 10 entre la valeur moyenne $\bar{I}_{\mathrm{I}}$ et la fluctuation qui permet le démarrage d'une oscillation dans le cas d'un cristal de $1 \mathrm{~cm}$.

Expériences avec des sondes non linéaires. - Les expériences déjà décrites posent plus de questions qu'elles n'en résolvent :
- Quelles dimensions ont les zones d'oscillation?

- Quelle durée ont leurs émissions?

- Quelles intensités $I_{\mathrm{S}}$ et $I_{\mathrm{L}}$ y règnent?

Pour y répondre, nous avons placé dans les champs rayonnés des éléments non linéaires capables d'émettre aux fréquences $2 \omega_{L}, \omega_{\mathrm{L}}+\omega_{\mathrm{S}}$ et $2 \omega_{\mathrm{S}}$ sous l'effet d'une excitation simultanée aux fréquences $\omega_{\mathrm{L}}$ et $\omega_{\mathrm{S}}$ et nous avons mesuré l'énergie ou la puissance des cinq ondes qui sont en jeu.

Pour exposer la méthode, supposons d'abord que nous analysons simplement une seule zone d'oscillation de surface $a^{2}$ où l'intensité excitatrice est $I_{\mathrm{L}}$ et dont l'intensité Stokes se maintient au niveau $I_{\mathrm{S}}$ pendant un temps $\tau$. Sur la surface irradiée $A^{2}$ règne en moyenne un carré d'intensité $\bar{I}_{\mathrm{L}}^{2}$.

Les longueurs d'onde laser et Stokes étant respectivement $6943 \AA$ et $7510 \AA$, une lame de quartz de $147 \mu$, perpendiculaire à l'axe optique, fabriquera avec une efficacité presque égale les trois fréquences de composition; leurs puissances seront :

$$
\begin{gathered}
p_{\mathrm{LL}}=k A^{2} \bar{I}_{\mathrm{L}}^{2} ; \quad p_{\mathrm{LS}}=k a^{2} I_{\mathrm{L}} I_{\mathrm{S}} ; \quad p_{\mathrm{SS}}=k a^{2} I_{\mathrm{S}}^{2} \\
\text { Par ailleurs, on mesure : } \\
p_{\mathrm{S}}=a^{2} I_{\mathrm{S}} \text { et } P_{\mathrm{L}}=A^{2} \bar{I}_{\mathrm{L}} .
\end{gathered}
$$

Le diagramme de rayonnement peut être différent pour chacune des trois fréquences de composition et il faut s'assurer que la collection des photons est aussi efficace dans les trois cas.

La mesure simultanée de $p_{\mathrm{LL}}$ et de $P_{\mathrm{L}}$, en l'absence de la calcite avec un temps de résolution $t$ trois fois plus court que la durée de l'impulsion $\omega_{L}$, donne une valeur de $k$, mais dans cette approximation on suppose vraie l'égalité :

$$
\left(\bar{I}_{\mathrm{L}}\right)^{2}=\frac{1}{A^{2} t} \iiint I_{\mathrm{L}}^{2}(x, y, t) \mathrm{d} x \mathrm{~d} y \mathrm{~d} t .
$$

L’appareil est alors « étalonné ».

Premier cas. - La durée d'émission $\tau$ est supérieure au temps de résolution $t$. On a alors trois équations $\left(p_{\mathrm{LS}}, p_{\mathrm{SS}}, p_{\mathrm{S}}\right)$ pour les trois inconnues ${ }^{7} a^{2}, I_{\mathrm{L}}$ et $I_{\mathrm{S}}$.

Deuxième cas : $\tau<t$. - Les valeurs crêtes $p^{\prime}$ mesurées par les détecteurs prennent alors une autre signification :

$p_{\mathrm{LS}}^{\prime}=k a^{2} \frac{\tau}{t} I_{\mathrm{L}} I_{\mathrm{S}} ; \quad p_{\mathrm{SS}}^{\prime}=k a^{2} \frac{\tau}{t} I_{\mathrm{S}}^{2} ; \quad p_{\mathrm{S}}^{\prime}=\frac{a^{2} \tau}{t} I_{\mathrm{S}}$.

On obtient alors $I_{\mathrm{S}}, I_{\mathrm{L}}$ et $\left(a^{2} \tau\right) / t$. Il faut alors une équation de plus pour analyser le produit $a^{2} \tau / t$. On peut tirer $a$ d'une mesure de divergence angulaire par la règle de diffraction.

Une fois a mesuré, la méthode permet de mesurer des temps $\tau$ très brefs avec des détecteurs très lents.

Gas général. - Pendant le temps de résolution $t$, il y a $N_{\mathrm{t}}$ émissions individuelles. Elles durent en 
moyenne $\tau$; leur surface est en moyenne $a^{2}$. C'est toujours la divergence qui donne l'ordre de grandeur de $a^{2}$.

Si le quartz est adhérant à une face de la calcite, les valeurs crêtes mesurées $P_{\mathrm{Ss}}, P_{\mathrm{LS}}$ et $P_{\mathrm{S}}$ donnent $N_{\mathrm{t}} \cdot a^{2} \tau / t, I_{\mathrm{L}}$ et $I_{\mathrm{S}} \cdot N_{\mathrm{t}} \tau / t$ est le nombre de zones émettant simultanément. On a comparé à $P_{\mathrm{ss}}$ la puissance $\Pi_{\mathrm{ss}}$ créée par la même puissance moyenne $P_{\mathrm{s}}$ dans un quartz placé, cette fois, à quelque distance de la calcite, au foyer d'une lentille de focale $f$.
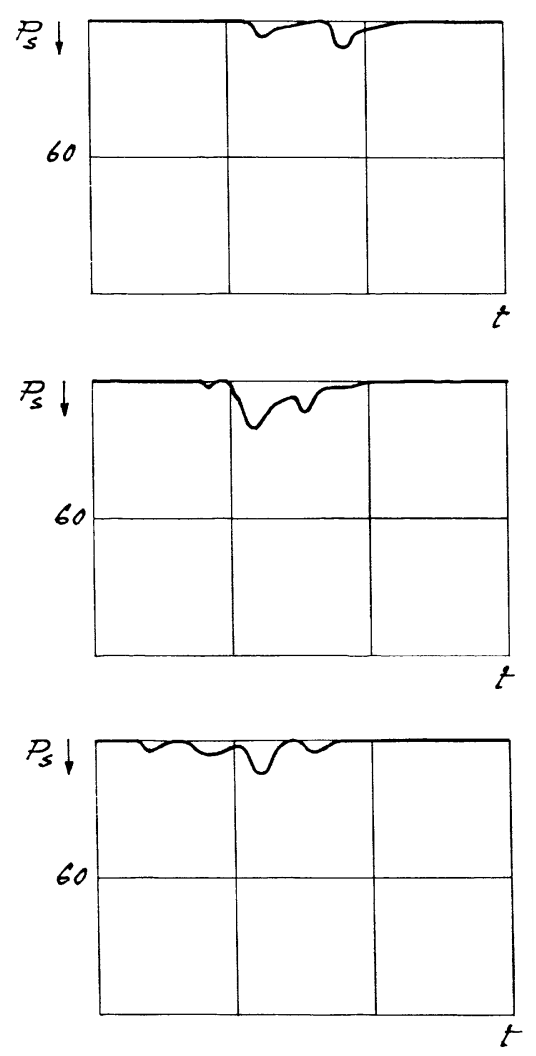

FiG. 6. - Raman du spath au niveau du kW : observation de $P_{\mathrm{S}}$ avec une résolution de $2 \times 10^{-9} \mathrm{~s}$. Temps : $10^{-8} \mathrm{~s}$ par carreau $-P_{\mathrm{s}}$ en $\mathrm{kW}$.
S'il n'y a pas de relations de phase entre les émissions simultanées, on obtient :

$$
\begin{array}{ll}
\Pi_{\mathrm{ss}}=P_{\mathrm{ss}} N_{\mathrm{t}} \frac{\tau}{t} \frac{a^{4}}{\lambda^{2} f^{2}} & \text { si } \quad N_{\mathrm{t}} \frac{\tau}{t}>1 \\
\Pi_{\mathrm{SS}}=P_{\mathrm{Ss}} \frac{a^{4}}{\lambda^{2} f^{2}} \quad \text { si } \quad N_{\mathrm{t}} \frac{\tau}{t}<1 .
\end{array}
$$

S'il y a relation de phase entre les diverses zones émettant simultanément, il vaut mieux les considérer comme une zone unique de plus grande surface.

La comparaison de $P_{\mathrm{LS}}$ et $\Pi_{\mathrm{LS}}$ est également intéressante. Ce genre d'expériences ne permet pas d'obtenir séparément $N_{\mathrm{t}}$ et $\tau$, donc de savoir si on observe un petit nombre de zones émettant longtemps ou un grand nombre d'émissions brèves.

L'observation directe de $P_{\mathrm{S}}$ avec un temps de résolution de $2 \times 10^{-9} \mathrm{~s}$ est possible pratiquement pour $P_{\mathrm{S}} \geqslant 1 \mathrm{~kW}$. De nombreux enregistrements analogues à ceux de la figure 6 suggèrent qu'à ces niveaux les émissions qui contribuent le plus à $P_{\mathrm{S}}$ durent souvent plus de $2 \times 10^{-9} \mathrm{~s}$.

Résultats expérimentaux relatifs aux sondes non linéaires. - Nous allons résumer les résultats acquis avec le dispositif de la figure $7 . P_{\mathrm{JLL}}, P_{\mathrm{LS}}$ et $P_{\mathrm{SS}}$ sont de l'ordre d'une fraction de milliwatt. Elles sont mesurées par un photomultiplicateur après sélection par des filtres ou des monochromateurs à prisme. Avec les monochromateurs, on est sûr de bien observer la fréquence souhaitée, mais on peut craindre les erreurs dues à la répartition spatiale éventuellement différente des ondes LL, LS et SS.

Avec les filtres, on est dans la situation inverse. Les quartz étaient placés soit en A (amont), soit en M (médian), soit en B (aval). Il pouvait aussi être éloigné de la calcite en amont ou en aval et être placé au foyer d'une lentille.

La plupart des mesures ont été faites sur des cristaux de 1 et $2 \mathrm{~cm}$ et sur un sandwich fait d'un quartz médian placé avec des joints optiques entre deux cristaux de $1 \mathrm{~cm}$.

L'excitation était faite par un faisceau laser rendu

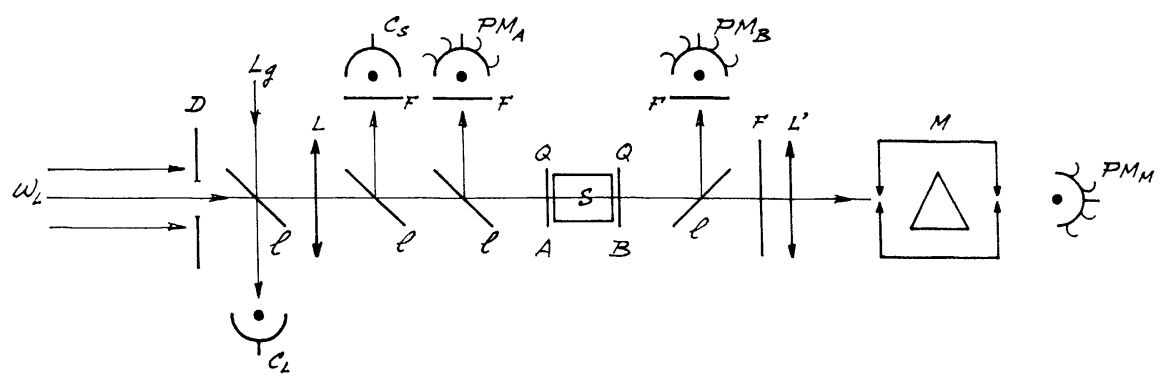

FIG. 7. - Dispositif de mesures avec des sondes non linéaires :

Q : Quartz de $147 \mu$. - S : Cristal de spath. — D : Diaphragme. - F : Filtres. - $\mathrm{C}_{\mathrm{L}}$ : Cellule pour $\omega_{\mathrm{J}}$. $C_{\mathrm{S}}$ : Cellule pour $\omega_{\mathrm{S}}-\mathrm{PM}_{\mathrm{A}}, \mathrm{PM}_{\mathrm{B}}, \mathrm{PM}_{\mathrm{M}}$ : Photomultiplicateurs pour $2 \omega_{\mathrm{L}}, \omega_{\mathrm{L}}+\omega_{\mathrm{S}}, 2 \omega_{\mathrm{S}}$. $-\mathrm{M}:$ Monochromateur. - $\mathrm{L}$ et $\mathrm{L}^{\prime}$ : Lentilles. 
convergent par une lentille de distance focale $60 \mathrm{~cm}$, la calcite étant en général placée à mi-chemin de la lentille et du foyer.

1. Quartz Placé en bout D'ÉGhantillon. - Dans le domaine $0,5 \mathrm{~kW}<P_{\mathrm{s}}<50 \mathrm{~kW}, P_{\mathrm{LS}}$ se montre proportionnelle à $P_{\mathrm{S}}$. D'après les équations (4) à (7) :

$$
\frac{P_{\mathrm{LS}}}{P_{\mathrm{I}, \mathrm{I}}} \simeq \frac{I_{\mathrm{L}}}{\bar{I}_{\mathrm{L}}} \cdot \frac{P_{\mathrm{S}}}{P_{\mathrm{L}}} .
$$

Il suffit d'une faible variation de $P_{\mathrm{I}}$, donc aussi de $P_{\mathrm{I} . \mathrm{L}}$, pour balayer ce domaine de $P_{\mathrm{S}}$; la proportionnalité observée était attendue. La valeur de sa constante nous renseigne sur le rapport de l'intensité $I_{\mathrm{L}}$ dans les zones d'émission, au moment où elles émettent, et de l'intensité moyenne $\bar{I}_{\mathrm{L}}$.

Les valeurs $I_{\mathrm{L}} / \bar{I}_{\mathrm{L}}$ ainsi obtenues vont de 16 à 30 .

Nous avons répété ces mesures avec de nombreuses petites variantes expérimentales (d'angles solides surtout) et $P_{\mathrm{LS}} / P_{\mathrm{LL}}$ reste toujours d'un ordre de grandeur supérieur à $P_{\mathrm{S}} / P_{\mathrm{L}}$.

La même anomalie $P_{\mathrm{LS}} / P_{\mathrm{LI}} \gg P_{\mathrm{S}} / P_{\mathrm{I}}$ est observée dans l'hydrogène excité par le même laser.

Il est difficile d'apprécier l'erreur probable qui affecte ces déterminations; elles reposent en effet sur une série assez longue de mesures et d'étalonnages d'une part, et sur l'incertaine équation (5) d'autre part.

$\mathrm{Si}$ on fait confiance à ces résultats, on y trouve une nouvelle indication que les oscillations prennent naissance dans des zones où $I_{\mathrm{L}}>\bar{I}_{\mathrm{L}}$.

Mais $P_{\mathrm{LL}}^{\mathrm{B}}$ créée dans un quartz placé en aval de la calcite (en B) est strictement égale à $P_{\mathrm{LL}}^{\mathrm{A}}$ créée dans un quartz amont (en A). Il n'y a donc pas d'indications que la calcite ait contribué à créer ces anomalies du champ laser. Mais nous n'avons pas encore fait $\left({ }^{1}\right)$ ce contrôle à très fort niveau de $P_{\mathrm{s}}$. La mesure directe de $P_{\text {LI }}$ est une méthode peu sensible de détection des anomalies de $I_{\mathrm{L}}$.

$P_{\mathrm{ss}}$ est, elle aussi, approximativement proportionnelle à $P_{\mathrm{s}}$ dans le domaine $0,5 \mathrm{~kW}<P_{\mathrm{s}}<10^{3} \mathrm{~kW}$. D'après les équations (4) à (7) :

$$
P_{\mathrm{SS}} / P_{\mathrm{L} \mathrm{S}}=I_{\mathrm{S}} / I_{\mathrm{L}}
$$

ce rapport reste voisin de $1 / 6$. Ce point est important; il confirme le concept de zones où, une fois le seuil d'oscillation franchi, $I_{\mathrm{S}}$ crôit jusqu'à être proche de $I_{\mathrm{L}}$.

$P_{\mathrm{Ss}}$ mesurée en arrière (quartz en $\mathrm{A}$ ) est égale à $P_{\mathrm{Ss}}$ mesurée en avant (quartz en B). Ce fait élimine la possibilité d'un effet de self-trapping analogue à celui qui, dans les liquides composés de molécules anisotropes, affecte également les ondes $\omega_{\mathrm{L}}$ et $\omega_{\mathrm{S}}$ en les concentrant vers l'avant.

Une comparaison directe, sans passer par les fentes d'un monochromateur, nous a donné $P_{\mathrm{SA}} \simeq P_{\mathrm{IL}}$ pour $P_{\mathrm{S}}=1,8 \mathrm{MW}$ et $P_{\mathrm{L}}=12 \mathrm{MW}$.

(1) De nouvelles mesures à fort nireau $\left(P_{\mathrm{S}} \simeq 0,3 P_{\mathrm{L}}\right)$ montrent encore $P_{\mathrm{I}, \mathrm{L}}^{\mathrm{B}} \leqslant P_{\mathrm{LL}}^{\mathrm{A}}$ et confirment l'absence de " self-focusing".
La comparaison de la puissance $\Pi_{s s}$ créée par $P_{\mathrm{s}}=1 \mathrm{~kW}$ dans un quartz placé au foyer d'une lentille $f=5 \mathrm{~cm}$ à la $P_{\mathrm{ss}}$ créée par la même $P_{\mathrm{s}}$ dans un quartz placé en $\mathrm{B}$ donne $\Pi_{\mathrm{ss}} / P_{\mathrm{Ss}} \simeq 1 / 3$. L'équation (7) assigne alors une valeur de $10^{-4} \mathrm{~cm}^{2}$ à la surface élémentaire $a^{2}$.

Une telle zone rayonnant une intensité de $50 \mathrm{MW} . \mathrm{cm}^{-2}$ par exemple émet une puissance de $5 \mathrm{~kW}$. Si une telle émission dure $10^{-9} \mathrm{~s}$ et est observée par un système ayant un temps de résolution de $10^{-8} \mathrm{~s}$, elle donnera une puissance crête apparente de $500 \mathrm{~W}$.

Nous vérifierons d'ailleurs que ces surfaces sont « rondes » en comparant les proportions de $P_{\mathrm{s}}$ qui passent dans une fente placée au foyer d'une lentille dans le cas où la fente est, soit parallèle, soit perpendiculaire au vecteur électrique.

L'équation (7) prédit que $\Pi_{\mathrm{Ss}} / P_{\mathrm{SS}}$ doit croître avec $P_{\mathrm{S}}$.

Si $P_{\mathrm{S}}$ croît parce que $N_{\mathrm{t}} \tau / t$ augmente, $a^{2}$ restant constante, $\Pi_{\mathrm{ss}} / P_{\mathrm{Ss}}$ doit croître comme $P_{\mathrm{S}}$.

Si $P_{\mathrm{S}}$ croît parce que, le nombre d'émetteurs restant constant, leur surface $a^{2}$ augmente, $\Pi_{\mathrm{Ss}} / P_{\mathrm{Ss}}$ doit croître comme $P_{\mathrm{s}}^{2}$. En fait, en faisant aller $P_{\mathrm{S}}$ de $25 \mathrm{~kW}$ à $1 \mathrm{MW}$, nous avons vu croître $\Pi_{\mathrm{Ss}} / P_{\mathrm{Ss}}$ d'un facteur 10 seulement. Ce fait est étonnant.

Peut-être les aberrations de notre lentille, pourtant très coûteuse, nous ont-elles empêchés d'observer les conséquences de l'augmentation de $a^{2}$ ? Nous poursuivons l'étude $\left({ }^{2}\right)$.

En suivant, à $P_{\mathrm{S}}$ constant, l'évolution de $P_{\mathrm{SS}}$ quand on éloigne le quartz de la calcite, nous avons vérifié en avant la valeur déjà trouvée pour $a$ par la méthode de la lentille. Mais en arrière la divergence mesurée par le «quartz éloigné » semble un peu plus grande qu'en avant.

2. Quartz mÉdian. - Nous avons étudié le système de la figure 8 . Les $P_{\mathrm{S}}$ émises en avant et en

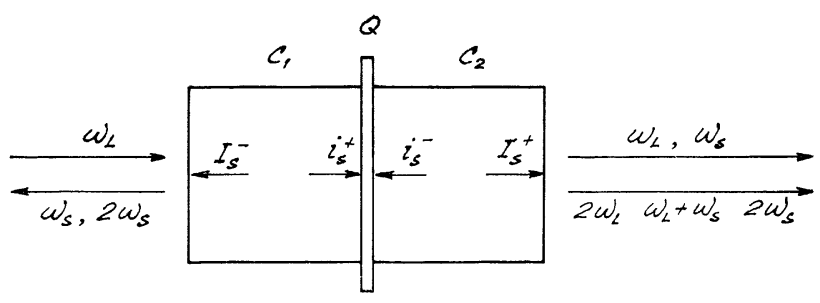

FIG. 8. - Sonde non linéaire en cavité :

$C_{1}$ et $C_{2}$ : Cristaux de $1 \mathrm{~cm}$. - Q : Quartz de $147 \mu$. $-\mathrm{C}_{1}, \mathrm{Q}$ et $\mathrm{C}_{2}$ sont collés à la glycérine.

$$
I_{\mathrm{s}}^{-}=I_{\mathrm{s}}^{+} ; \quad I_{\mathrm{s}}^{-}=G_{1} i_{\mathrm{s}}^{-} ; \quad I_{\mathrm{s}}^{+}=G_{2} i_{\mathrm{s}}^{+} \text {. }
$$

arrière sont toujours égales. Les oscillations résonnent donc bien sur les faces terminales (on pouvait craindre

$\left({ }^{2}\right)$ Les résultats expérimentaux cités ici sont corrects, ils signifient que, même à fort niveau, l'ensemble des zones d'émission de $P_{\mathrm{S}}$ ressemble à une touffe d'épingles toujours pointues. 
qu'une calcite fonctionne en oscillatrice et l'autre en amplificatrice).

Nous avions entrepris cette étude dans l'espoir de mettre en évidence une éventuelle augmentation du pouvoir réflecteur des bouts de la cavité Stokes; cette anomalie aurait évidemment facilité l'accrochage ou l'entretien des oscillations; elle aurait augmenté le rapport de la puissance $P_{\mathrm{Ss}}$ créée dans le quartz médian et de la puissance $P_{\mathrm{S}}$ mesurée à l'extérieur de cette cavité devenue bonne.

En fait, nous n'avons rien observé de tel (d'autres expériences relatives à des cavités munies de miroirs très dissymétriques nous ont confirmé qu'il n'y avait pas de « miroirs magiques »).

Dans cette configuration, pour une même $P_{\mathrm{s}}$ mesurée à l'extérieur, les intensités $i_{\mathrm{S}}^{+}$(vers l'avant) et $i_{\mathrm{S}}^{-}$(vers l'arrière) sont moindres au niveau du quartz médian que les intensités $I_{\mathrm{S}}^{+}$et $I_{\mathrm{S}}^{-}$que voient des quartz placés aux extrémités. Ignorons les autres sources de pertes ou supposons-les égales pour les ondes + et les ondes -; l'égalité des pouvoirs réflecteurs assure l'égalité de $I_{\mathrm{S}}^{+}$et $I_{\mathrm{s}}^{-}$. Mais il ne s'ensuit pas que $i_{\mathrm{S}}^{+}=i_{\mathrm{S}}^{-}$.

$P_{\mathrm{Ss}}$ mesurée en arrière vaut $1 / 3$ de $P_{\mathrm{ss}}$ mesurée en avant. Appelons $G_{1}$ et $G_{2}$ les gains respectifs du premier et du second cristal ( $f g .8$ ) et supposons constante la section de l'onde $\omega_{\mathrm{S}}$ le long de la cavité, nous concluons :

$$
G_{1}=\sqrt{3} G_{2} \text {. }
$$

L'intensité $I_{\mathrm{L}}$ s'affaiblirait un peu le long de la cavité. Le rapport $P_{\mathrm{ss}} / P_{\mathrm{S}}$ mesuré vers l'avant est 8 à 10 fois plus faible et le rapport $P_{\mathrm{LS}} / P_{\mathrm{S}}$ trois fois plus faible que si le quartz était en bout de cavité. On peut en conclure $G_{2}=3$.

Pour clore ce paragraphe consacré à l'utilisation des sondes non linéaires, nous voulons mentionner un phénomène qui peut perturber ce genre de mesures : l'onde Stokes concentrée par une lentille de focale $7 \mathrm{~cm}$ sur la fente métallique ouverte à $10^{-2} \mathrm{~cm}$ de l'entrée d'un monochromateur, le vecteur électrique étant perpendiculaire à la fente, y produit une certaine puissance lumineuse à la fréquence double.

Pour $P_{\mathrm{s}} \simeq 100 \mathrm{~kW}$, on recueille à la sortie $\mathrm{du}$ monochromateur $p_{\mathrm{SS}} \simeq 10^{-4} \mathrm{~W}$.

Les mesures du seuil. - Nous avons mesuré le seuil ${ }^{*} \bar{I}_{\mathrm{L}}^{0}$ d'intensité moyenne excitatrice $\bar{I}_{\mathrm{I}}$ au-delà duquel se produisent les effets stimulés forts dans des cristaux de diverses longueurs munis de faces plus ou moins réfléchissantes.

Le laser fonctionne toujours à la même puissance; on fait varier $\bar{I}_{\mathrm{L}}$ en atténuant avec des verres gris. On peut espérer ainsi garder inchangé le rapport entre les plus fortes fluctuations $I_{\mathrm{L}}$ et la valeur moyenne $\bar{I}_{\mathrm{I}}$.

Une condition d'oscillation s'écrit de la façon la plus simple : l'amplification due à l'effet stimulé compense les pertes de la cavité résonnante. Mais deux traits particuliers aux présentes expériences en compliquent un peu l'analyse :

a) Les pertes par diffraction ne peuvent être négligées dans des zones de petit diamètre;

b) L'intensité $I_{\mathrm{L}}$ et le gain $G$ qui en résultant varient dans le temps.

Les pertes par diffraction. - Plusieurs des expériences décrites (divergence de $\omega_{\mathrm{S}}$, mesures simultanées de fortes $I_{\mathrm{S}}$ et de faibles $P_{\mathrm{S}}$, faible valeur de $\Pi_{\mathrm{Ss}}$ créée dans le quartz placé au foyer d'une lentille) indiquent que le diamètre $a_{\mathrm{S}}$ des zones de création de $\omega_{\mathrm{s}}$ est de l'ordre de $10^{-2} \mathrm{~cm}$. On peut imaginer que le diamètre $a_{\mathrm{L}}$ des inhomogénéités du champ laser, qui leur permettent de prendre naissance, est lui aussi assez petit.

Schématisons le problème : supposons l'existence de zones où l'intensité $I_{\mathrm{L}}$ est répartie selon une symétrie de révolution autour d'un axe confondu avec la direction de propagation et la normale aux faces terminales du cristal; soit $a_{\mathrm{L}}$ son « diamètre à demi-amplitude »:

- Quel sera alors le diamètre $a_{\mathrm{s}} \mathrm{du}$ mode Stokes qui pourra osciller?

- Quelles seront les pertes par diffraction de ce mode?

Dans les calculs de Fox et Li, les diaphragmes sont localisés en bout de cavité et le milieu amplificateur est uniforme. On peut alors calculer les répartitions de champ $E_{\mathrm{i}}(x, y, z)$ de chaque mode, et leurs pertes, en considérant uniquement la cavité passive.

Pour nous, c'est la forme du milieu actif qui joue le rôle d'un « diaphragme continu » et contribue finalement avec les faces à imposer une certaine répartition $E(x, y, z)$ avec laquelle les gains par stimulation pourront compenser les pertes par diffraction et transmission.

Si $a_{\mathrm{S}}<a_{\mathrm{L}}$, le mode Stokes profite au mieux des forts gains, mais les pertes par diffraction s'en trouvent accrues. De fortes pertes par transmission sur les faces terminales contribuent à réduire $a_{\mathrm{S}}$.

Pour obtenir une solution plus précise, il faudrait adopter la méthode de Fox et Li en demandant à la machine de tenir compte de la forme du milieu amplificateur. Dans l'opérateur de Huyghens qui associe la répartition de champ du plan $z+b$ à celle du plan $z$, il faudrait appliquer le facteur de gain relatif au rayon vecteur considéré. Le choix de la distance d'itération $b$ devra être fait relativement aux quantités $a_{\mathrm{L}}^{2} / \lambda$ et $1 / \beta$ où $\beta$ désigne l'indice de gain sur l'axe de $I_{\mathrm{L}}$.

Sans pouvoir d'inventer la réponse de la machine, nous proposons quelques relations dimensionnelles : quand l'inégalité $\beta \gg \lambda / a_{\mathrm{L}}^{2}$ est satisfaite, l'idée du « diaphragme continu » prend une réalité physique; c'est précisément alors que pour le calcul des pertes de $\omega_{s}$ nous proposons de le remplacer par des dia- 
phragmes discrets à intervalles $b$ choisis tels qu'on ait simultanément :

$$
b \lambda / a^{2}<1 \quad \text { et } \quad \beta b>1 .
$$

La fraction $\Delta I_{\mathrm{S}}$ de l'énergie $I_{\mathrm{S}}$ présente sur un diaphragme qui sera éliminée au diaphragme suivant (c'est-à-dire perdue pour le circuit d'amplification) vaudra alors :

$$
\Delta I_{\mathrm{S}}=-I_{\mathrm{S}} b \lambda / a_{\mathrm{S}}^{2} .
$$

Tout se passera comme si les pertes par diffractions pouvaient se représenter par un indice d'atténuation :

$$
\alpha_{\mathrm{D}}=\lambda / a_{\mathrm{S}}^{2}
$$

et la condition d'oscillation sera, compte non tenu des autres pertes :

$$
\beta>\lambda / a_{\mathrm{S}}^{2}
$$

avec $\lambda=5 \times 10^{-5} \mathrm{~cm}$ et $a_{\mathrm{S}}=10^{-2}$, cela donne $\beta>0,5 \mathrm{~cm}^{-1}$.

Cinétique. - Une fois franchi le seuil $I_{\mathrm{L}}^{0}$ défini par l'égalité du gain et des pertes, il faut que l'inégalité $I_{\mathrm{L}}>I_{\mathrm{I}}^{0}$ se maintienne assez franchement et longtemps pour que le produit $a_{\mathrm{S}}^{2} I_{\mathrm{S}}$ atteigne une puissance décelable.

Prenons l'exemple d'un cristal de $2 \mathrm{~cm}$ avec faces dans l'air. Le seuil $I_{\mathrm{I}}^{0}$ correspondra, en chaque parcours qui dure $10^{-10} \mathrm{~s}$, à la compensation des pertes par diffraction qu'on peut estimer à $\mathrm{e}^{\mathbf{1}}$ et des pertes par transmission qui valent $\mathrm{e}^{\mathbf{3}}$.

Si $I_{\mathrm{L}}=1,25 I_{\mathrm{L}}^{0}$ pendant $10^{-9} \mathrm{~s}, I_{\mathrm{S}}$ croît de $\mathrm{e}^{10}$ au-dessus de son niveau spontané. Si $I_{\mathrm{L}}=1,5 I_{\mathrm{L}}^{0}$ pendant $10^{-9} \mathrm{~s}, I_{\mathrm{S}}$ croîtra de $\mathrm{e}^{20}$.

Plus faibles sont les pertes, plus fort doit être le rapport $I_{\mathrm{L}} / I_{\mathrm{L}}^{0}$ pendant un temps donné pour que $I_{\mathrm{S}}$ atteigne un niveau détectable.
Résultats expérimentaux. - La figure 9 montre les valeurs d'intensité moyenne mesurées au seuil de cristaux de différentes longueurs avec leurs faces dans l'air $(R=6 \%)$ ou dans l'eau $(R=1 \%)$. Ces mesures ont été faites en lumière convergente. La figure 4 nous rappelle que même une mesure précise de $\bar{I}_{\mathrm{L}}$ ne mène pas à une détermination de $\overline{I_{\mathrm{L}}^{0}}$ avec une précision relative meilleure que $20 \%$.

Les échantillons de $0,5 \mathrm{~cm}$ montrent une valeur de seuil instable quand on ne contrôle pas la température ambiante. Leurs modes longitudinaux sont espacés de $0,6 \mathrm{~cm}^{-1}$ alors que la raie spontanée est large de $1,1 \mathrm{~cm}^{-1}$. Cette instabilité provient peut-être de ce que le mode de la cavité le mieux placé est plus ou moins loin du pic de la raie. Le point relatif au cristal de $0,5 \mathrm{~cm}$ qui est sur la figure 9 est choisi parmi les points les plus bas.

Lorsque l'échantillon est mis « de travers », le seuil est plus élevé. Si, par exemple, on éloigne de $30 \mathrm{mrd}$ la normale des faces d'un cristal de $2 \mathrm{~cm}$ de l'axe du faisceau laser, le seuil remonte d'un facteur 2. Cet effet étaye l'hypothèse de surintensité de $I_{\mathrm{L}}$ dans des zones de forme allongée.

Si on suit l'idée trop simple qu'au seuil le gain compensera les seules pertes par transmission, on est étonné de la relative insensibilité des seuils aux longueurs des cristaux et aux pouvoirs réflecteurs de leurs faces.

En multipliant par 16 la longueur, on ne divise le seuil que par 4.

En noyant dans l'eau un cristal de $1 \mathrm{~cm}$ ou de $8 \mathrm{~cm}$, on ne voit remonter les seuils que du facteur 1,3 et non 1,6 .

En munissant un cristal de $8 \mathrm{~cm}$ de deux miroirs

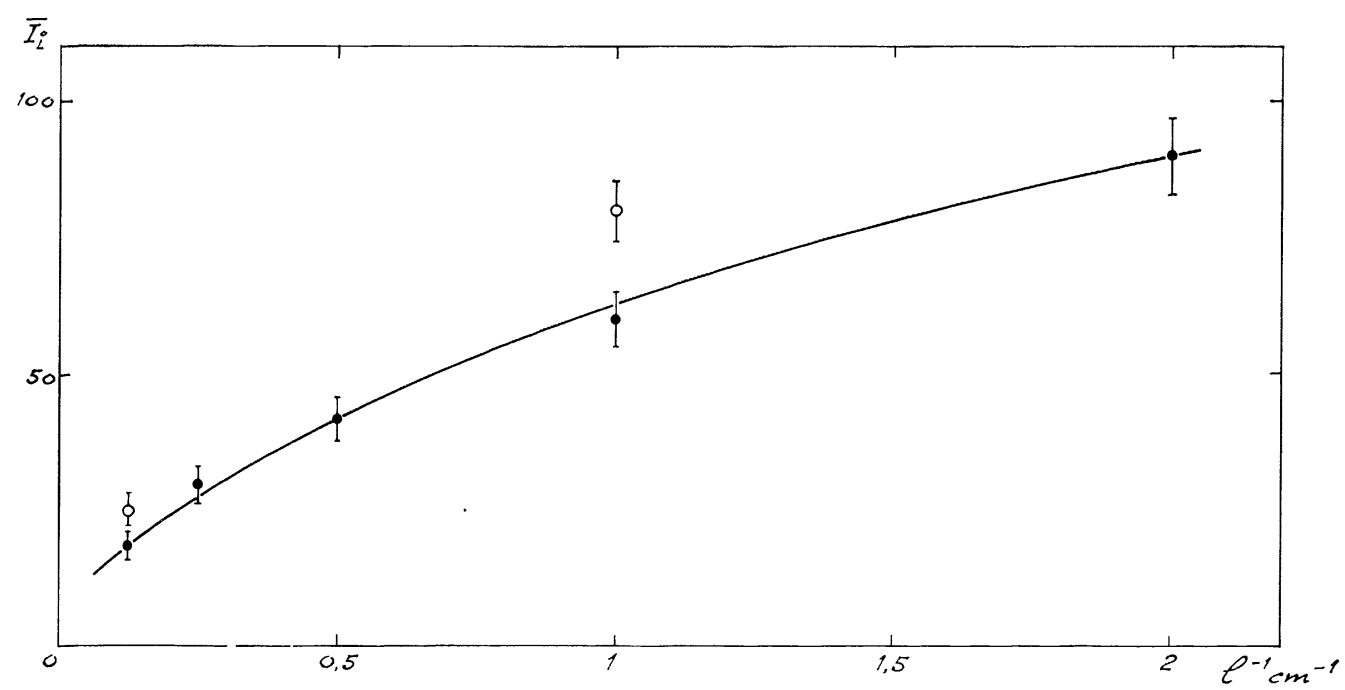

FIG. 9. - Seuils de cristaux de diverses longueurs :

$I_{\mathrm{L}}^{0}$ : Intensité moyenne en $\mathrm{mW} / \mathrm{cm}^{-2}$. - - - : Faces terminales dans l'air ; -o- : Faces dans l'eau. Lumière convergente; cristaux au foyer d'une lentille de focale de $60 \mathrm{~cm}$. - Abscisses : Inverse de la longueur. 
ayant $70 \%$ de réflexions, le seuil ne baisse que du facteur 0,75 .

En introduisant dans le bilan les pertes par diffraction, en faisant appel aux considérations cinétiques, on peut atténuer le désaccord entre ce qu'on calcule et ce qu'on mesure, mais il persiste.

Dans un cristal de $1 \mathrm{~cm}$, l'influence des pertes par diffraction est sans doute faible; de même le « retard à la détection » dû à la cinétique de croissance de l'oscillation d'un cristal aussi court doit être négligeable; aussi peut-on s'étonner de la faiblesse du rapport des seuils mesurés dans l'air et dans l'eau. Interprété directement, ce rapport de 1,3 suggère que l'indice de gain varie comme $I_{\mathrm{L}}^{2}$ plutôt que comme $I_{\mathrm{L}}$.

Nous avons aussi utilisé des cristaux à faces non parallèles en complétant la cavité par un miroir extérieur « aval ». Le seuil remonte un peu quand le miroir est éloigné du cristal. Un cristal oblique de $2 \mathrm{~cm}$ muni d'un miroir $(R=6 \%)$ placé à $5 \mathrm{~cm}$ en aval montre un seuil 1,4 fois plus élevé qu'un cristal de $2 \mathrm{~cm}$ à faces parallèles. Les cavités longues sont donc « défavorisées ».

Mesures spatialement bien localísées. - Nous avons tenté de nous affranchir de l'irritante incertitude relative aux rapports $I_{\mathrm{L}} / \bar{I}_{\mathrm{L}}$ en diaphragmant très sévèrement le faisceau excitateur et en le mesurant avec une résolution de $2 \times 10^{-9} \mathrm{~s}$.

L'expérience a été faite avec un cristal de $4 \mathrm{~cm}$ placé à $2 \mathrm{~cm}$ en arrière d'un diaphragme de $400 \mu$. Le seuil $I_{\mathrm{L}}^{0}$ observé dans ces conditions est de $75 \mathrm{MW} . \mathrm{cm}^{-2}$; il est supérieur d'un facteur 2,5 au seuil $\bar{I}_{\mathrm{L}}^{0}$ d'intensité moyenne mesurée sur un diaphragme de $3 \mathrm{~mm}$.

Pour cette valeur $I_{\mathrm{L}}^{0}$, le gain calculé du cristal vaut $\mathrm{e}^{1,32}=3,7$; c'est encore insuffisant pour expliquer l'oscillation. Cette mesure confirme l'analyse spatio-temporelle du faisceau laser que nous avons présentée au début (cf. page 98).

La fluctuation $I_{\mathrm{L}}=3 \bar{I}_{\mathrm{L}}$ est certainement présente, mais la fluctuation $I_{\mathrm{L}}=10 \bar{I}_{\mathrm{L}}$ qui serait nécessaire à l'oscillation des cristaux courts n'est pas mise en évidence.

De toute façon, mesurer des seuils reste une méthode trop indirecte de mesurer des gains; nous avons essayé de les mesurer directement.

Mesures d'amplification. - MEsures en Gavité. La comparaison des puissances $P_{\mathrm{S}}$ reçues simultanément par les quatre cellules du dispositif de la figure 10 permet de définir un gain dans chacun des deux cristaux qui constituent la cavité Stokes.

Par exemple, avec $l_{\mathrm{a}}=1 \mathrm{~cm}$ et $l_{\mathrm{b}}=2 \mathrm{~cm}$, on trouve $\quad G_{\mathrm{a}}=P_{\mathrm{S}}^{2} / P_{\mathrm{s}}^{1}=1,5 ; \quad G_{\mathrm{b}}=P_{\mathrm{S}}^{4} / P_{\mathrm{s}}^{3}=2,4 \quad$ et $P_{\mathrm{S}}^{1}=P_{\mathrm{s}}^{4}$ pour des $P_{\mathrm{S}}^{\mathrm{i}}$ de l'ordre de $10 \mathrm{~kW}$.

Les gains mesurés ainsi sont nettement insuffisants pour justifier les oscillations $\omega_{\mathrm{S}}$ puisque $G_{\mathrm{A}} G_{\mathrm{B}}$ n'atteint pas 16. On peut l'expliquer ainsi : une bonne partie de la lumière Stokes qui atteint les cellules a été

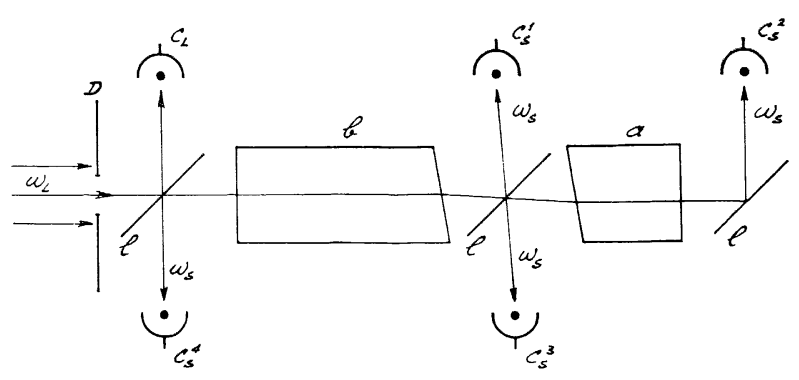

FIG. 10. - Mesures de gain en cavité :

a et $\mathrm{b}$ : Cristaux à faces obliques. $-\mathrm{C}_{\mathrm{s}}^{1}, \mathrm{C}_{\mathrm{s}}^{2}, \mathrm{C}_{\mathrm{s}}^{3}, \mathrm{C}_{\mathrm{s}}^{4}$ : Cellules mesurant $\omega_{\mathrm{s}}$. Diverses combinaisons de longueur de $a$ et $b$ ont été utilisées.

diffractée dans la cavité hors des zones où $I_{\mathrm{L}}$ est grande et n'a pas profité des grands gains sur toute la longueur des cristaux. L'éloignement des deux cristaux joue un rôle dans cet effet (voir fig. 13).

Mesures hors cavité. - La figure 11 montre le dispositif. Le gain $G$ est défini par le rapport des

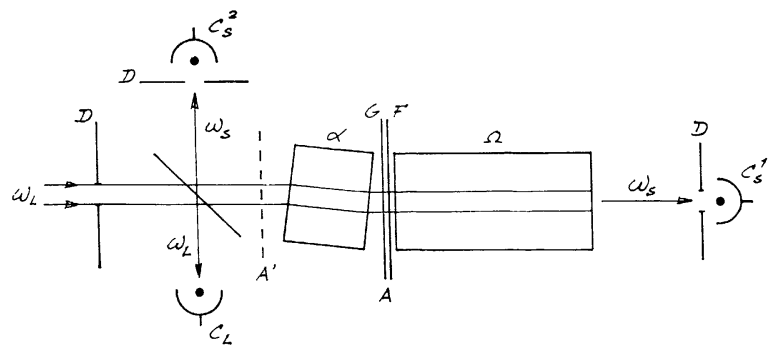

FIG. 11. - Mesures de gain hors cavité :

$\Omega$ : Cristal oscillateur. $-\alpha$ : Cristal amplificateur (faces parallèles ou obliques). - G : Verre gris transportable de $A$ en $A^{\prime}$. - $F$ : verre $B G 36$ transparent pour $\omega_{\mathrm{L}}$, absorbant pour $\omega_{\mathrm{S}} .-\mathrm{C}_{\mathrm{S}}^{1}, \mathrm{C}_{\mathrm{S}}^{2}$ : Cellules pour $\omega_{\mathrm{S}}$.

quotients $P_{2} / P_{1}$ avec et sans cristal amplificateur. Un verre assez transparent pour $\omega_{\mathrm{L}}$ et assez opaque pour $\omega_{\mathrm{S}}$ est indispensable entre cristaux oscillateur et amplificateur. En effet, $I_{\mathrm{S}}$ n'est que peu inférieure à $I_{\mathrm{L}}$ à la sortie de l'oscillateur; $I_{\mathrm{L}}$ ne pourrait plus guère l'amplifier dans l'amplificateur sans être ellemême atténuée; ainsi atténuée en amont de l'oscillateur, elle ne pourrait plus l'entretenir. Un amplificateur amont ne peut donc fonctionner à grand gain $G$ en même temps que l'oscillateur si on n'interpose pas entre eux un verre de transmission telle que $T_{\mathrm{S}} G \ll 1$.

Tant que cette condition est satisfaite, nous avons vérifié que dans un large domaine (4 ordres de grandeur) $G$ était indépendant de la puissance Stokes présente à l'entrée de l'amplificateur.

Un verre gris transporté de A en $\mathrm{A}^{\prime}$ permet d'étudier l'inıluence de $I_{\mathrm{I}}$ sur l'amplificateur sans modifier le fonctionnement de l'oscillateur. 
On évite l'entrée en oscillation des amplificateurs longs en les disposant de travers ou en les munissant de faces non parallèles. Cette dernière disposition empêche aussi les rayons ayant subi plusieurs parcours dans l'amplificateur d'atteindre le détecteur.

La figure 12 donne quelques résultats dans le cas

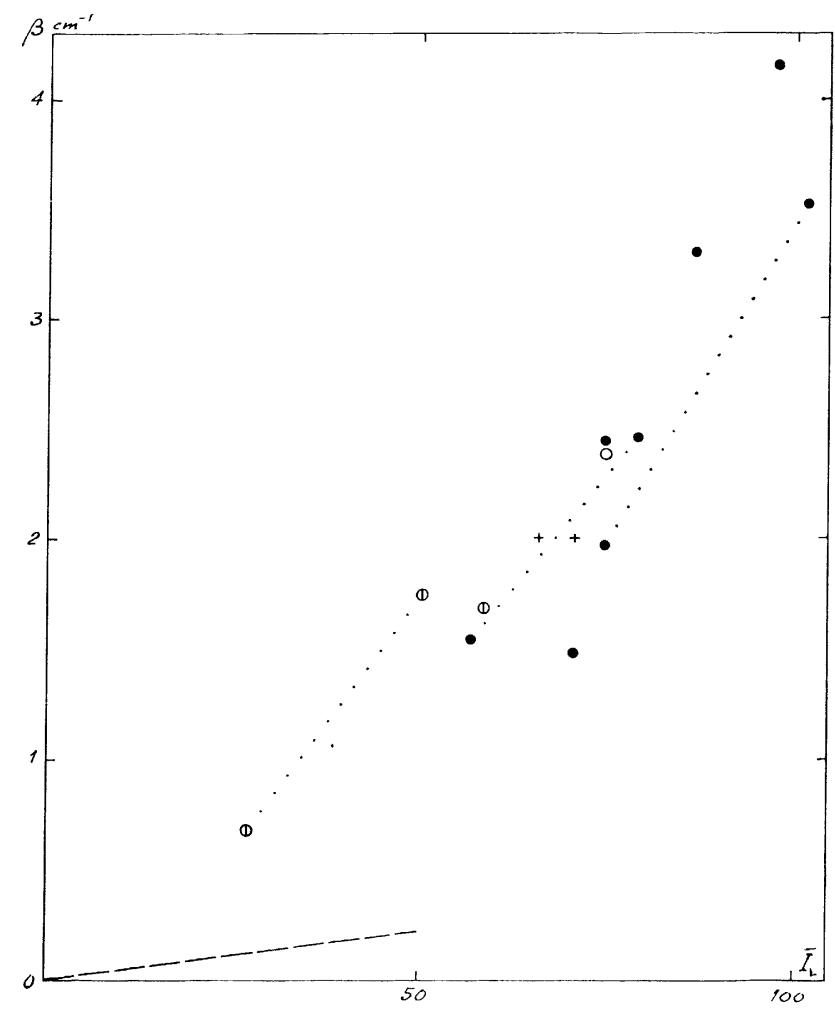

FI's. 12. - Indices d'amplification $\beta$ en $\mathrm{cm}^{-1}$ en fonction de l'intensité moyenne $\bar{I}_{\mathrm{L}}$ en $\mathrm{MW} . \mathrm{cm}^{-2}$ :

I umière convergente. - Distance entre amplificateur et oscillateur $0,3 \mathrm{~cm}$ (de face à face).

$+: \beta$ mesurée sur des cristaux de $0,2 \mathrm{~cm}$.

- : $\beta$ mesurés sur des cristaux de $0,5 \mathrm{~cm}$.

- : $\beta$ mesurés sur des cristaux de $1 \mathrm{~cm}$.

(1) : $\beta$ mesurés en face d'un autre laser sur des cristaux de $1 \mathrm{~cm}$.

Les paires de points liés par une ligne pointillée sont obtenus par transport du verre gris (cf. texte). La droite tracée de l'origine correspond à $\beta=b \bar{I}_{\mathrm{L}}$ avec $b=4,4 \times 10^{-3} \mathrm{~cm} \cdot \mathrm{MW}^{-1}$. Cristaux $\Omega$ de 1 à $4 \mathrm{~cm}$.

où les deux cristaux sont très proches $(d=0,3 \mathrm{~cm})$. La figure 13 montre l'effet de la distance entre cristaux oscillateur et amplificateur. Chaque point représente la moyenne d'une trentaine de mesures :

1) Les indices de gain mesurés en fonction de $\bar{I}_{\mathrm{L}}$ ne suffisent pas à justifier les oscillations, mais ils sont nettement plus grands que ceux qu'on mesure « en cavité »;

2) Les gains croissent très vite quand on rapproche les deux cristaux. En se risquant à extrapoler vers $d \rightarrow 0$

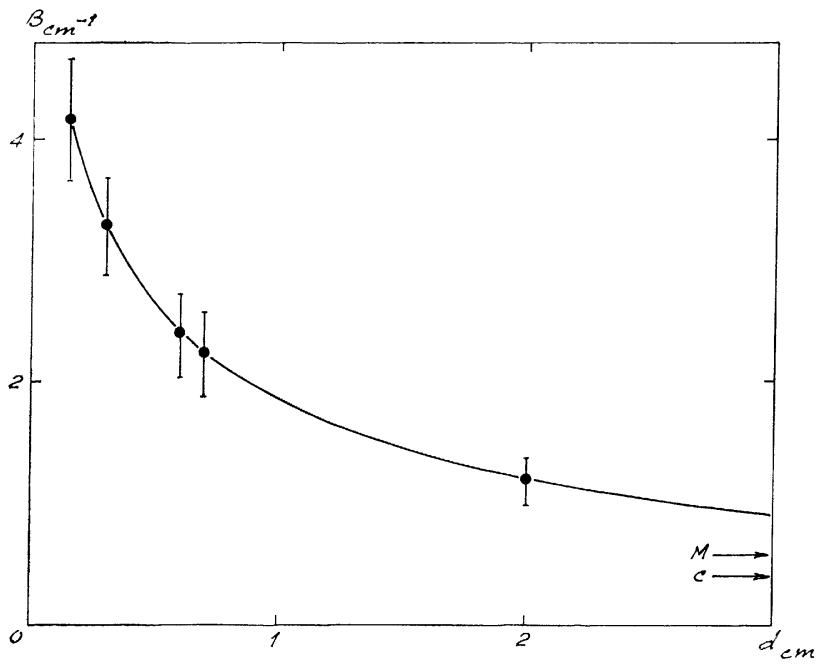

FIG. 13. - Indice de gain $\beta$ en $\mathrm{cm}^{-1}$ en fonction de la distance entre cristaux amplificateur et oscillateur : Amplificateur de $0,5 \mathrm{~cm}$. - Intensité moyenne $\bar{I}_{\mathrm{L}}=90 \mathrm{MW} \cdot \mathrm{cm}^{-2}$. - Ordonnée de la flèche $\mathrm{M}$ : $\beta$ mesuré à $30 \mathrm{~cm}$ de l'oscillateur. - Ordonnée de la flcèhe $C: \beta$ calculé ; $\beta=4,4 \times 10^{-3} \bar{I}_{\mathrm{L}}$

la courbe $\beta(d)$, on trouve des valeurs de $\beta$ qui justifient à peu près les oscillations;

3) Malgré cet effet, le logarithme du gain semble proportionnel à la longueur de l'amplificateur dans le domaine $0,2 \mathrm{~cm}-2 \mathrm{~cm}$;

4) A grande distance $(d=30 \mathrm{~cm})$, les gains mesurés sont très faibles. Ils ont bien une valeur de l'ordre de celle que prédit l'équation fondamentale (1);

5) La présence d'un amplificateur long ne diminue pas le seuil d'oscillation du deuxième cristal.

Tous ces effets confirment qualitativement le concept de zones d'oscillation étroites axées sur des zones de grandes $I_{\mathrm{L}}$ très étroites aussi; la rapide décroissance des gains mesurés avec la distance signifie alors que les «diamètres » $a_{\mathrm{L}}$ et $a_{\mathrm{S}}$ sont de l'ordre de $10^{-2} \mathrm{~cm}$. La comparaison des points 2) et 3) suggère que, dans la calcite, mais non dans l'air, les zones de grandes $I_{\mathrm{L}}$ « retiennent » l'onde $\omega_{\mathrm{s}}$. Le point 5) confirme que la calcite ne contribue pas à créer ces zones de grandes $I_{\mathrm{L}}$

6) La figure 12 montre aussi que l'indice de gain ne varie pas comme $\bar{I}_{\mathrm{L}}$ mais plutôt comme $\bar{I}_{\mathrm{L}}^{2}$ pour les fortes $\bar{I}_{\mathrm{L}}$.

Ge point est nouveau et nous voulons préciser comment il est établi. Soit par exemple un cristal dont le gain mesuré vaut $\mathrm{e}^{3}=20,1$ en présence du faisceau direct du laser, un verre gris de transmission $T_{\mathrm{L}}=0,5$ étant placé en $\mathrm{B}$. Transportons ce verre en $\mathrm{B}^{\prime}$, le gain tombe à 4,5 si $\beta$ varie comme $I_{\mathrm{L}}$, à 2,1 si $\beta$ varie comme $I_{\mathrm{L}}^{2}$. En transportant un verre $T_{\mathrm{L}}=0,7, G$ tombe à 8,2 si $\beta$ varie en $I_{\mathrm{L}}$ et à 4,5 si $\beta$ varie en $I_{\mathrm{I}}^{2}$. La différence des chiffres prédits par 
les deux lois est nettement plus grande que les erreurs de mesure.

Les expériences répétées avec des couples oscillateuramplificateur de longueurs variées sont plutôt en faveur de la loi $I_{\mathrm{I}}^{2}$.

Si on diminue $I_{\mathrm{L}}$ à un niveau tel que $\beta l<1$, la différence des chiffres prédits par les deux lois se rapproche des erreurs de mesure de cette méthode.

Les gains ainsi mesurés à chaque expérience sont en fait des moyennes puisque les rayons qui traversent le cristal n'y subissent pas tous la même amplification. Le jeu des moyennes agissant par l'intermédiaire de la fonction exponentielle a pour effet d'augmenter apparemment la puissance de $I_{\mathrm{L}}$ qui agit sur l'indice $\beta$ mesuré par cette méthode. Nous voulons dire que, si $\beta$ dépendait vraiment de $I_{\mathrm{L}}$, c'est une dépendance envers une puissance de $I_{\mathrm{L}}$ supérieure à 1 qui apparaîtrait dans nos expériences. Mais il est difficile d'imaginer des situations où ce jeu de moyennes ferait apparaître une dépendance de $\beta$ envers $I_{\mathrm{L}}$ aussi éloignée de la loi linéaire.

La loi $\beta\left(I_{\mathrm{L}}\right)$. - Des résultats expérimentaux relatifs aux effets Raman stimulés dans l'hydrogène ont déjà mis en évidence que $\beta$ semblait y varier comme $I_{\mathrm{L}}^{2}$ plutôt que comme $I_{\mathrm{L}}(\mathbf{3})$.

Pour expliquer cet effet dans la calcite, nous avons envisagé plusieurs hypothèses.

Dispersion ANORMALE. - Une anomalie d'indice de réfraction est associée au pouvoir amplificateur dû à l'effet Raman stimulé; l'indice est augmenté pour les fréquences comprises dans la moitié supérieure de la raie spontanée. Pour $\omega \simeq \omega_{\mathrm{L}}-\omega_{\mathrm{v}}+\frac{\Delta \omega_{\mathrm{S}}}{2}$, cet accroissement d'indice $\Delta n$ est maximum et $\Delta n \simeq \beta_{\mathrm{m}} \lambda_{\mathrm{s}} / 4 \pi$ où $\beta_{m}$ désigne le gain au pic de la raie.

Si l'intensité excitatrice est concentrée en filaments étroits, comme le suggèrent beaucoup d'effets expérimentaux, le gradient d'indice qui en résultera sur leurs bords tendra à concentrer les ondes Stokes telles que $\omega>\omega_{\mathrm{L}}-\omega_{\mathrm{v}}$ vers les régions de grandes $I_{\mathrm{L}}$.

Cet effet compensera efficacement les effets de la diffraction quand on aura :

$$
\Delta n=\beta_{\mathrm{m}} \lambda_{\mathrm{s}} / 4 \pi>\lambda^{2} / a_{\mathrm{s}}^{2} .
$$

Nous reconnaissons l'équation (8) établie plus haut d'un point de vue différent. $I_{\mathrm{L}}$ aurait alors un double rôle : créer le gain et maintenir l'onde Stokes dans les régions de grand gain. La dépendance des indices de gain mesurés envers $I_{\mathrm{L}}$ serait alors plus forte que celle d'une simple loi linéaire. Mais pour profiter de cette concentration, l'onde Stokes devra démarrer à une fréquence $\omega \neq \omega_{\mathrm{L}}-\omega_{\mathrm{v}}$ et ne bénéficiera pas du gain optimum $\beta_{m}\left(\omega_{L}-\omega_{v}\right)$.

$\left({ }^{3}\right)$ Effects of Phonon lifetime on stimulated optical scattering in gases, E. E. HAGENLOCKER, R. W. Minck, W. G. RADO, Ford Scientific Laboratory Report, août 1966 .
La mise en évidence expérimentale d'un excès systématique de $\omega_{\mathrm{s}}$ sur la valeur $\omega_{\mathrm{L}}-\omega_{\mathrm{v}}$ pose de difficiles problèmes.

EfFets Raman D'ordre supérieur. - Si la loi $\beta\left(I_{\mathrm{L}}\right)$ révélait en rayonnement stimulé un mécanisme plus complexe que le simple effet Raman du premier ordre, on devrait pouvoir mettre en évidence le phénomène spontané correspondant.

Cette réflexion nous ramène à notre point de départ, à savoir l'absence de courbure constatée au pied des courbes $P_{\mathrm{S}}\left(\bar{I}_{\mathrm{L}}\right)$.

Cette absence est déjà gênante dans le cadre de la théorie la plus simple et nous oblige à imaginer d'assez fortes fluctuations de $I_{\mathrm{L}}$. Elle est également en contradiction avec l'hypothèse d'un effet en $I_{\mathrm{L}}^{2}$ par exemple, mais on peut faire deux remarques : d'une part, l'éventuel effet en $I_{\mathrm{L}}^{2}$ serait de toute façon dilué dans l'effet spontané en $I_{\mathrm{L}}$; d'autre part, une fluctuation locale de $I_{\mathrm{L}}$ amplifiera beaucoup plus nettement les effets en $I_{\mathrm{I}}^{2}$.

L'acte élémentaire de l'effet simple en $I_{\mathrm{I}}$ peut être décrit par le schéma :

$$
\omega_{\mathrm{L}}+M \rightarrow M^{*}+\omega_{\mathrm{S}} .
$$

Un photon $\omega_{\mathrm{L}}$ agit sur la molécule $M$ et disparaît en lui donnant un quantum vibrationnel et en créant un photon $\omega_{\mathrm{s}}$.

Quel pourrait être le mécanisme d'une loi en $I_{\mathrm{L}}^{2}$ ?

- Création simultanée de Stokes et d'anti-Stokes :

$$
2 \omega_{\mathrm{L}}+M \rightarrow M+\omega_{\mathrm{S}}+\omega_{\mathrm{AS}}
$$

Nous avons mesuré le rapport des puissances $P_{\mathrm{AS}} / P_{\mathrm{S}}$ dans des échantillons de diverses longueurs $l$ pour $\operatorname{des} P_{\mathrm{S}}$ allant de 1 à $10 \mathrm{~kW}$ :

$$
\begin{array}{lll}
P_{\mathrm{As}} / P_{\mathrm{S}}=5 \times 10^{-2} & \text { pour } & l=0,5 \mathrm{~cm} \\
P_{\mathrm{AS}} / P_{\mathrm{S}}=10^{-2} & \text { pour } & l=1 \mathrm{~cm} \\
P_{\mathrm{AS}} / P_{\mathrm{S}}<5 \times 10^{-3} & \text { pour } & l=2 \mathrm{~cm} .
\end{array}
$$

Ces résultats excluent le schéma (B) de création simultanée de $\omega_{\mathrm{S}}$ et $\omega_{\mathrm{AS}}$. Ils sont au contraire en accord avec le principe d'une onde $\omega_{\mathrm{AS}}$ se construisant aux dépens de l'onde $\omega_{\mathrm{L}}$ grâce à une onde $\omega_{\mathrm{S}}$ créée indépendamment.

- Contribution du deuxième Stokes : Dans un échantillon de $1 \mathrm{~cm}$, pour $P_{\mathrm{s}} \simeq 1 \mathrm{~kW}$, on mesure $P_{\mathrm{S}_{2}} / P_{\mathrm{S}_{1}} \simeq 5 \times 10^{-4}$; cette faible valeur signifie que $\omega_{\mathrm{S}}$ n'intervient pas dans le démarrage de $\omega_{\mathrm{s}}$.

- Excitation simultanée de paires proches de molécules :

$$
2 \omega_{\mathrm{L}}+2 M \rightarrow 2 M^{*}+2 \omega_{\mathrm{s}} .
$$

Ge schéma prévoit pour $\omega_{\mathrm{S}}$ un indice de gain proportionnel à $I_{\mathrm{L}}^{2}$ d'une part et à $I_{\mathrm{S}}$ d'autre part. Or nous avons vérifié que le gain pour l'onde $\omega_{\mathrm{s}}$ ne croissait pas avec le niveau d'entrée de l'amplificateur. 
- Effet à quatre photons :

$$
2 \omega_{L}+M \rightarrow M^{*}+\omega_{L}+\omega_{S} .
$$

De même qu'une transition électronique peut s'effectuer par l'absorption simultanée de deux photons, on peut concevoir un effet Raman à quatre photons. Le schéma (D) conduirait d'ailleurs à une dépendance de l'indice de gain $\beta$ en $I_{\mathrm{L}}^{3}$. La section efficace probable de ce type de transitions nous paraît bien faible pour nous tirer d'affaire.

Conclusion. - La validité du concept de gain pour l'onde $\omega_{\mathrm{S}}$ nous paraît solidement établi par l'ensemble des expériences.

Nous avons obtenu des indications numériques sur le diamètre des zones d'oscillation et sur l'intensité Stokes qui y règne.

Les oscillations naissent dans des endroits où, par instants, l'intensité $I_{\mathrm{L}}$ dépasse notablement l'intensité moyenne $\bar{I}_{\mathrm{L}}$.

L'observation de forts indices de gain $\beta$ dans des cristaux courts en amont du cristal oscillateur montre que ces fluctuations $I_{\mathrm{L}}$ ne sont pas dues à des anomalies de propagation de $\omega_{\mathrm{L}}$ dans la calcite.
Les expériences menées avec une bonne résolution spatio-temporelle indiquent : $I_{\mathrm{L}}<4 \bar{I}_{\mathrm{L}}$.

Pour expliquer dans le cadre de la théorie la plus simple l'ordre de grandeur des gains mesurés, il faudrait $I_{\mathrm{L}} \simeq 10 \bar{I}_{\mathrm{L}}$.

Les sondes non linéaires « voient » $I_{\mathrm{L}} \simeq 20 \bar{I}_{\mathrm{L}}$, cette mesure étant faite en aval des zones d'oscillation au moment où $I_{\mathrm{S}}$ est proche de $I_{\mathrm{L}}$. La différence entre ces trois facteurs numériques est déjà assez préoccupante, mais c'est la forme non linéaire de la fonction $\beta\left(I_{\mathrm{L}}\right)$ qui pose le problème le plus difficile.

Le rôle de la dispersion anomale est sans doute important. Des expériences analogues à celles que nous avons décrites sont actuellement menées avec un laser oscillant sur un seul mode $\mathrm{TEM}_{00}$ pour éclaircir ces questions.

Remerciements. - Nous remercions la Direction des Recherches et Moyens d'Essais de son constant soutien matériel et moral. Nous sommes reconnaissants envers François Gires de nous avoir aidés de toutes les ressources de son esprit subtil et critique à chaque étape du présent travail.

\section{BIBLIOGRAPHIE}

Einstein (A.), EHrenfest (P.), Z. Physik, 1923, 19, 301.

Heliwarth (R. W.), Applied Optics, 1963, 2, 847.

Bloembergen (N.), Shen (Y. R.), Phys. Rev. Lett., 1964, 12, 504, et Quantum Electronics, Porto Rico, 1965.

ECKHARD'T (G.), BORTFELD (D. P.), Geller (M.), $A p p l$. Phys. Lett., 1963, 3, 137.
Chiao (R.), Stoicheff (B. P.), Phys. Rev. Lett., 1964, $12,290$.

Giordmaine (J. A.), KaIser (W.), Phys. Rev., 1966, 144, 676.

PARK (K.), Physics Lett., 1966, 22, 39.

Bisson (G.), Mayer (G.), C. R. Acad. Sc., 1967, 265, 397. 